\title{
LABORATORY-BASED BIDIRECTIONAL REFLECTANCE DISTRIBUTION FUNCTIONS OF RADIOMETRIC TARPS
}

\author{
Georgi T. Georgiev", James J. Butler ${ }^{\mathrm{b}}$ \\ ${ }^{a}$ Science Systems and Applications, Inc., Lanham, MD 20706, e-mail: \\ georgi.t.georgiev@nasa.gov \\ ${ }^{b}$ NASA Goddard Space Flight Center, Code 614.4, Greenbelt, MD 20771
}

\begin{abstract}
Laboratory-based bidirectional reflectance distribution functions of radiometric tarp samples used in the vicarious calibration of Earth remote sensing satellite instruments are presented in this paper. The results illustrate the BRDF dependence on the orientation of the tarps' weft and warp threads. The study was performed using the GSFC scatterometer at incident zenith angles of $0^{\circ}, 10^{\circ}$, and $30^{\circ}$; scatter zenith angles from $0^{\circ}$ to $60^{\circ}$; and scatter azimuth angles of $0^{\circ}, 45^{\circ}, 90^{\circ}$, $135^{\circ}$ and $180^{\circ}$. The wavelengths were $485 \mathrm{~nm}, 550 \mathrm{~nm}, 633 \mathrm{~nm}$ and $800 \mathrm{~nm}$. The tarp's weft and warp dependence on BRDF is well defined at all measurement geometries and wavelengths. The BRDF difference can be as high as $8 \%$ at $0^{\circ}$ incident angle and $12 \%$ at $30^{\circ}$ incident angle. The fitted BRDF data shows a very small discrepancy from the measured ones. New data on the forward and backscatter properties of radiometric tarps are reported. The backward scatter is well pronounced for the white samples. The black sample has well pronounced forward scatter. The provided BRDF characterization of radiometric tarps is an excellent reference for anyone interested in using tarps for radiometric calibrations. The results are NIST traceable.
\end{abstract}

Keywords: Bidirectional Reflectance Distribution Function, Optical instrumentation and measurements, Metrology, Reflectance spectroscopy. 


\section{INTRODUCTION}

The ability to monitor, understand, and predict the Earth's climate and environmental processes depends on the quality of data from Earth's remote sensing instruments. The global nature of Earth's processes requires consistent long-term calibration of all instruments involved in data retrieval ${ }^{1}$. The bidirectional reflectance distribution function (BRDF) defines the directional reflection characteristics of an optical surface. It provides the reflectance of a target in a specific direction as a function of illumination and viewing geometry. The BRDF is a function of wavelength and reflects the structural and optical properties of the surface. Various space and airborne radiometric and imaging remote sensing instruments use diffuse scatter plates as calibration sources requiring preflight BRDF calibration measurements ${ }^{2,3}$. On-board diffusers are used to trend on-orbit instrument radiance or reflectance calibration. Laboratory based diffusers are used for pre-flight instrument radiance calibrations. BRDF measurements of natural targets are also used for remote sensing characterization of vegetation canopies and soils ${ }^{4}$, oceans ${ }^{5}$, or especially large pollution sources ${ }^{6}$.

The data reported in this study are intended to more completely describe the BRDF of radiometric tarp samples with particular interest on the effect of tarp weft and warp treads orientation. This study was done in support of the commercial remote sensing radiometric characterization program of IKONOS multispectral imagery at NASA's Stennis Space Center, from which the samples were obtained ${ }^{7,8}$. The samples are witness pieces from larger chemically treated field-deployed radiometric canvas tarps used as reference reflectance standards in remote sensing characterizations. The radiometric tarps were also used to perform spatial characterizations measuring the sensors ability to image an edge formed by using two contrasting tarps. The tarps are manufactured with strict specifications; they are large enough to characterize the spatial characteristics of $1 \mathrm{~m}$ ground sample distance (GSD) class imagery. The targets can easily be deployed over alternative sites; the proper care at deployment being of great importance. The accuracy of such tarp-based field calibrations depends on an accurate 
knowledge of the tarps laboratory measured BRDF at the different geometries of source and scatter zenith and azimuth angles. The reported data is traceable to NIST's Special Tri-function Automated Reference Reflectometer (STARR) ${ }^{9}$ via test standards.

\section{BRDF BACKGROUND}

The BRDF definition and derivation are credited to Nicodemus et al. ${ }^{10}$ who examined the problem of defining and measuring the scatter of diffuse and specular optical materials. Following his concept the scatter defining geometry is shown in Fig.1, where the subscripts $i$ and $s$ refer to incident and scatter quantities, respectively. He also assumed that all scatter comes from the sample surface and none from the bulk. He defined the BRDF in radiometric terms as the ratio of the surface radiance $L_{s}$ scattered by a surface into the direction $\left(\theta_{\mathrm{s}}, \phi_{\mathrm{s}}\right)$ to the incident surface irradiance $E_{i}$ incident on a unit area of the surface at a particular wavelength:

$$
B R D F=\frac{d L_{s}\left(\theta_{i}, \phi_{i}, \theta_{s}, \phi_{s} ; E_{i}\right)}{d E_{i}\left(\theta_{i}, \phi_{i}\right)},
$$

where the subscripts $i$ and $s$ denote incident and scattered respectively, $\theta$ the zenith, and $\phi$ the azimuth angles.

Nicodemus further assumed that the beam has a uniform cross section, the illuminated area on the sample is isotropic, and all scatter comes from the sample surface. In practice, we are dealing with real samples' surfaces which are not isotropic and the optical beams used to measure the reflectance are not perfectly uniform. Hence from the practical considerations the BRDF can be defined, as presented by Stover $^{11}$, as the scattered power per unit solid angle normalized by the incident power and the cosine of the detector zenith angle. It is expressed in terms of incident power, scattered power and the geometry of incident and reflected light:

$$
B R D F=\frac{P_{s} / \Omega}{P_{i} \cos \theta_{s}},
$$


where $P_{\mathrm{i}}$ is the incident power, $P_{\mathrm{s}}$ is the scatter power, $\theta_{\mathrm{s}}$ is the detector zenith angle and $\Omega$ is the solid angle determined by the area of detector aperture, $A$, and the distance from the sample surface to the limiting aperture at the detector assembly, $R$, or $\Omega=\mathrm{A} / \mathrm{R}^{2}$.

We are using the above BRDF expression (Eq.2) as it allows for bulk scatter in addition to surface scatter and permits non-uniform incident beam profiles. BRDF has units of inverse steradians and can range from very small numbers (e.g. off-specular black samples) to very large values (e.g. highly reflective samples at specular reflectance). The BRDF is often called cosinecorrected, when the $\cos \theta_{s}$ factor is not included. We are following the Nicodemus - Stover convention through this study; however care is required as some publications do not follow it. For example, although the remote sensing community follows the Nicodemus approach as a complete description of the BRDF of a surface, Eq.1, it prefers to use van de Hulst defined Bidirectional Reflection Function (BRF) in dealing with actual measurements under natural conditions:

$$
B R F=\frac{\pi I_{\lambda}\left(\theta, \theta_{0}, \Phi\right)}{\mu_{0} F_{\lambda}},
$$

where $I_{\lambda}$ is a reflected intensity, $F_{\lambda}$ is the solar flux density incident on the top of the atmosphere, $\theta$ and $\theta_{0}$ are respectively the scatter zenith and incident zenith angles, $\Phi$ is the azimuth angle between the scatter and incident light directions and $\mu_{0}=\cos \theta_{0}$. The BRF is dimensionless, normalized to the reference illumination irradiance.

\section{MEASUREMENT SETUP AND SAMPLES}

The radiometric tarp samples were studied in the Diffuser Calibration Facility at NASA's Goddard Space Flight Center (GSFC) using the facility's scatterometer. The scatterometer, located in a class 10000 laminar flow cleanroom, is capable of measuring the BRDF or bidirectional transmissive distribution function (BTDF) of a wide range of sample types including white diffusers, gray-scale diffusers, black painted or anodized diffusers, polished or 
roughened metal surfaces, clean or contaminated mirrors, transmissive diffusers, liquids, and granular solids. The operational spectral range of the instrument is from $230 \mathrm{~nm}$ to $900 \mathrm{~nm}$. The scatterometer facilitates computerized measurements at selected incident and scattered geometries and wavelengths for complete data acquisition. The measurement uncertainty, $\Delta_{\mathrm{BRDF}}$, was subsequently evaluated by Schiff et al. ${ }^{13}$ and Cady et al. ${ }^{14}$

$$
\left(\Delta_{\text {BRDF }}\right)^{2}=2\left(\Delta_{N S}\right)^{2}+2\left(\Delta_{\text {LIN }}\right)^{2}+\left(\Delta_{S L D}\right)^{2}+\left(\Delta_{\theta s} \tan \left(\theta_{s}\right)\right)^{2},
$$

It depends on several instrument variables. It was consequently evaluated in accordance with NIST guidelines ${ }^{12}$ to be less than $1 \%$ standard uncertainty. The main sources of uncertainty are 1) signal to noise ratio; 2) nonlinearity of the detector and electronics; 3) receiver solid angle; and 4) the total scatter angle error. The main sources of error are considered independent. Schiff et al. ${ }^{13}$ presents the detailed study on measurement uncertainty.

The samples in this study are small witness samples of the large tarps used in the vicarious calibration efforts of Stennis Space Center. Three measurements were performed at each wavelength, scatter geometry, and polarization state of the incident light and the average is reported. The measured data was found to be consistent to within $0.5 \%$. The high accuracy laboratory BRDF is provided to serve as a reference to the field observations and to deal with all wrinkle and terrain roughness.

The scatterometer can perform in-plane and out-of-plane BRDF and BTDF measurements, and $8^{\circ}$ directional/hemispherical measurements. It consists of a vertical optical source table, a sample stage, a detector goniometer, and a computer system for positioning control, data collection and analysis. Fig.2 shows the instrument's optical layout. The optical table can be rotated around its horizontal axis located at the table center to change the incident angle, $\theta_{\mathrm{i}}$, relative to the sample normal. The optical source table contains two possible light sources - a 75 W xenon short-arc lamp coupled to a Chromex 250SM scanning monochromator and a $\mathrm{He} / \mathrm{Ne}$ laser. Although not shown on Fig.2, additional laser sources are possible. The xenon lamp assembly is compact and generates an output beam focused on a monochromator entrance slit. A 
filter wheel is attached to the exit slit to block higher order spectra. Spherical and flat mirrors focus the output monochromatic beam on a fixed aperture. The optical beam is then directed to the sample surface by one spherical and two flat mirrors. The incident light is linearly polarized by a Glan-Thompson polarizer. The optical path for the laser source is simpler. Flat mirrors direct the laser beam to the sample. The incident beam is collimated by two lenses and linearly polarized by a different Glan-Thompson polarizer. When measurements are made with the xenon short-arc lamp source the folding mirror for the laser beam, shown in Fig.2 with dashed line, which would block the xenon lamp beam, is removed.

Fig. 3 shows the goniometer mechanism of the scatterometer. The scattered light from the sample is collected using an ultraviolet-enhanced silicon photodiode detector with output fed to a computer-controlled lock-in amplifier. The sample is mounted on a stage in the horizontal plane. The sample stage allows proper positioning of the sample with respect to the incident beam. It can be moved in $\mathrm{X}, \mathrm{Y}$ and $\mathrm{Z}$ linear directions using three motors. The sample stage provides sample rotation in the horizontal plane around the $\mathrm{Z}$ axis enabling changes in the incident azimuth angle, $\varphi_{\mathrm{i}}$. Sample stage leveling is adjustable using two manual micrometers. Various holders are available to support samples of different sizes, shapes, and thicknesses. Samples can be as large as $45 \mathrm{~cm}$ square and up to $4.5 \mathrm{~kg}$ in weight. However, larger and heavier samples can be measured by using an appropriate external sample stage.

The position of the detector assembly is determined by the scatter zenith and scatter azimuth angles. The detector assembly can be rotated around the vertical, Z, and horizontal, X, Y axes of the goniometer. As shown in Fig. 3 the detector moves along the arc providing the ability to make scatter measurements as a function of the scatter zenith angle, $\theta_{\mathrm{s}}$. The arc rotates $180^{\circ}$ around the vertical $\mathrm{Z}$ axis which determines the scatter azimuth angle, $\varphi_{\mathrm{s}}$. The center of the illuminated spot on the surface of the sample must be positioned at the cross point of the three perpendicular goniometer rotation axes, $\mathrm{X}, \mathrm{Y}, \mathrm{Z}$, coinciding with the center of a sphere with radius equal to the distance between that point and the detector's assembly cover aperture. 
The operation of the scatterometer is fully computerized. Custom software was developed to control all motion, data acquisition, and data analysis. The optical beams are mechanically chopped and a lock-in data acquisition technique is used. The electronics consists of a motion control module and a lock-in-amplifier. A custom pre-amp embedded into the detector housing was also designed and built.

The Diffuse Calibration Facility has participated in several round-robin ${ }^{14}$ measurement campaigns with domestic and foreign institutions. It has supported a number of NASA and international remote sensing Earth and space projects. The facility has characterized many types of samples including Spectralon ${ }^{15}$, Aluminum diffusers, Barium Sulfate, optical elements, Martian regolith simulant ${ }^{16}$, natural samples as vegetation and soil ${ }^{17}$, etc.

\section{EXPERIMENT}

The four tarp samples being studied are all $10 \mathrm{~cm} \times 10.5 \mathrm{~cm}$ cut along the weft and warp thread directions. The warp and weft are weaving terms. The warp is the set of lengthwise yarns across which the weft is shuttled back and forth to create a woven fabric. The samples are of similar material structure, possessing a highly regular wave-like structure but with different reflectance. The surface structure of the samples is shown on a microscopic image of tarp sample 1 in Fig.4. The tarps were made of woven polyester duck with a protective silicone pigmented coating. The samples were mounted flat on the scatterometer sample stage. The illuminated area depends on the incident angle. It varies from $16 \mathrm{~mm}$ in diameter at $0^{\circ}$ incident angle to an ellipse with a long axis of $28 \mathrm{~mm}$ at $30^{\circ}$ incident angle.

The bidirectional reflectance distribution of any sample strongly depends on the direction of the incident light on the sample and the detector's position as determined by the scatter zenith and scatter azimuth angles. The incident light along the sample normal describes the sample's bidirectional reflectance distribution at normal incidence while the incident light at angles other 
than normal describes the bidirectional reflectance distribution at non-normal incidence. The incident and scatter angles of this study were chosen by Stennis Space Center based on the illumination geometries of their vicarious airborne calibrations. Both in-the-principal-plane and out-of-the-principal-plane scans were performed hereafter referred to as in-plane and out-ofplane. The incident angles (i.e. source zenith) were $0^{\circ}, 10^{\circ}$, and $30^{\circ}$; the source azimuth angle was fixed at $4^{\circ}$. The latter is a result of IKONOS orbital mechanics and the need to have a tilted edge that can help to create an edge response relatively free from aliasing effects. The in-plane scans are at scatter azimuth angles of $0^{\circ}$, and $180^{\circ}$. The out-of-plan scans were at scatter azimuth angles of $45^{\circ}, 90^{\circ}$, and $135^{\circ}$. All the scans were performed at scatter zenith angles from $0^{\circ}$ to $60^{\circ}$ in $5^{\circ}$ steps. The wavelengths were 485, 550, 633 and $800 \mathrm{~nm}$ with a spectral bandwidth of $12 \mathrm{~nm}$. The detector field-of-view was centered on the samples for all measurements. We used the underfilled BRDF measurement technique meaning the illuminated area on the sample was always smaller than the detector FOV. All measurements in the current study were made for polarizations of the incident beam both parallel and perpendicular to the plane of incidence. The BRDF values for both polarizations were then averaged to yield the BRDF for unpolarized incident radiant flux, and the values of the unpolarized scattering case are reported in this paper.

The $8^{\circ}$ directional/hemispherical reflectance of the same samples was also measured. The $8^{\circ}$ integrating sphere is a separate accessory to the scatterometer. The sphere collects and spatially integrates the scattered optical radiation. The sphere interior is Spectralon with a typical reflectance of $94 \%$ to $99 \%$ from the UV to the NIR. The sphere was designed with four ports to accommodate the sample, the detector, and the entry of the incident light. A fourth port is a spare and is typically closed using a Spectralon plug. The total port area is less than $5 \%$ of the total surface area of the sphere. It is important to have the radiation balance established inside the sphere after as few internal reflections as possible. The light intensity incident on the detector should correspond to the average light intensity inside the sphere. The sphere is provided with an 
interior baffle to assure that the detector will not view radiation reflected directly from the sample.

\section{RESULTS \& DISCUSSION}

The analysis of radiometric tarp samples BRDF is presented for normal and non-normal angles of incidence. In addition, the tarps $8^{\circ}$ directional/hemispherical reflectance is given in Table 7. The measured BRDF has been fitted using a fifth-degree polynomial regression:

$$
\mathrm{Y}=\mathrm{A}+\mathrm{Bx}+\mathrm{Cx^{2 }}+\mathrm{Dx}{ }^{3}+\mathrm{Ex}^{4}+\mathrm{Fx}^{5}
$$

where $\mathrm{X}$ is the scatter zenith angle or $\Theta_{\mathrm{s}}, \mathrm{Y}$ is the $\operatorname{BRDF}\left(\Theta_{\mathrm{s}}\right)$, and $\mathrm{A}, \mathrm{B}, \mathrm{C}, \mathrm{D}, \mathrm{E}, \mathrm{F}$ are the polynomial coefficients. The coefficients, A, B, C, D, E, and F, given in Tables 1 to 3, were calculated at $485 \mathrm{~nm}$ wavelength, scatter azimuth angles of $0^{\circ}, 45^{\circ}, 90^{\circ}, 135^{\circ}, 180^{\circ}$, incident angles of $0^{\circ}, 10^{\circ}$ and $30^{\circ}$, and scatter zenith angles from $-60^{\circ}$ to $60^{\circ}$. The polynomial regression can be used for deriving BRDF data at random scatter zenith angles, $\Theta_{\mathrm{s}}$, for the above-mentioned scatter azimuth and incident angles at $485 \mathrm{~nm}$. The performance was evaluated by the RootMean-Square Error (RMSE), the most commonly used measure of success of numeric prediction. The error has the same dimensions as the predicted values themselves. The RMSE criterion is:

$$
\sigma=\sqrt{\frac{1}{N} \sum\left(f^{\prime}-f\right)^{2}},
$$

where $f$ ' is the modeled, $f$ is the measured BRDF and $\mathrm{N}$ is the number of points measured.

The tarps were studied first at normal incidence, the BRDF was measured at $0^{\circ}, 45^{\circ}, 90^{\circ}$, $135^{\circ}$, and $180^{\circ}$ scatter azimuth angles. The scatter zenith angles vary from $10^{\circ}$ to $60^{\circ}$ in $5^{\circ}$ steps. The scatter zenith angle of $0^{\circ}$ was not measured as at this geometry the detector is obscured by the last fold mirror. The BRDF at normal incidence of all tarp samples at a wavelength of 485 $\mathrm{nm}$ is shown in Fig.5 for scatter azimuth angles $0^{\circ}$ and $180^{\circ}$. The scatter zenith angles at $0^{\circ}$ scatter azimuth angle are presented in this figure as negative in order to be in accordance with 
the standard angular convention. In the following discussions, we will refer only to sample 1 , as there are not substantial differences between the samples except their absolute reflectance.

The BRDF of sample 1 at $485 \mathrm{~nm}$ and the study scatter azimuth angles is shown in Fig.6 as a function of scatter zenith angle at normal incidence illumination. The measurements at 550,633 and $800 \mathrm{~nm}$ are not represented as they show the same tendencies and would be redundant. Two types of data are presented in Fig. 6 - the fitted BRDF as plotted from the polynomial coefficients in Table 1, and the measured data points. The RMSE criterion has been used to calculate the error between the fitted and experimental data. The values for the study scatter azimuth angles, both normal and non-normal incidence are given in Table 4 for sample 1 at $485 \mathrm{~nm}$. The largest error at normal incidence is $2.32 \times 10^{-4}$.

The surfaces of the tarp samples are structured; therefore even at normal incidence their BRDF depends on the weft and warp thread orientation. The scatter from the surface depends mainly on the scatter zenith angle. However, the warp bumps obscure the detector from viewing the full surface. Therefore the scatter also depends on the scatter azimuth angle whether the detector scan plane is perpendicular or not to the weft threads, Fig.4. The BRDF is higher where the weft threads lie parallel to the detector scan plane; $\mathrm{X}-\mathrm{Z}$ plane as then the obscuration is minimal. The maximum obscuration occurs where the detector scan plane is parallel to the warp bumps, corresponding to the lower BRDF; Y-Z plane. The obscuration effect depends on the scatter zenith angle also. It is higher at larger scatter zenith angles. In addition to the obscuration, a shadowing effect takes place at non-normal incident angles. Both the obscuration and shadowing effects are shown schematically in Fig.7. The highest BRDF of tarp 1 was recorded at $0^{\circ}$ and $180^{\circ}$ scatter azimuth where the tarp weft threads lie parallel to the detector scan plane. The BRDF decreases at $45^{\circ}$ and $135^{\circ}$ scatter azimuth angles as the detector rotates to an orientation $45^{\circ}$ relative to the sample warp bumps. The lowest BRDF was measured at $90^{\circ}$ scatter azimuth, in accordance with our expectations. 
The data in Table 5 show what differences the observer should expect for airborne measurements depending on the scatter zenith angle assuming that the source (Sun) is at zenith. The data in this table represents the deviation in the tarp optical scattering from Lambertian. To make the comparison simpler we designate the BRDF at $30^{\circ}$ scatter zenith as a reference and compare the BRDF at other scatter zenith angles to the $30^{\circ}$ value. The BRDF differences were calculated from the BRDF data of sample 1 at $485 \mathrm{~nm}$, normal incidence, and scatter azimuth angles of $0^{\circ}, 45^{\circ}, 90^{\circ}, 135^{\circ}$ and $180^{\circ}$. It was found that the difference could be as high as $13 \%$ for a $5^{\circ}$ scatter zenith angle. The BRDF difference on the scatter zenith angle decreases as the wavelength increases. For example, the difference between BRDF at $30^{\circ}$ and $5^{\circ}$ scatter zenith is $13.02 \%$ at $485 \mathrm{~nm}$ and $8.83 \%$ at $800 \mathrm{~nm}$. The difference between BRDF at $30^{\circ}$ and $60^{\circ}$ scatter zenith is $-6.65 \%$ at $485 \mathrm{~nm}$ increasing to $2.76 \%$ at $800 \mathrm{~nm}$.

The dependence of BRDF on wavelength at normal incidence for sample 1 is given in Fig. 8 at 485, 550, 633 and $800 \mathrm{~nm}$ and at scatter azimuths of $0^{\circ}$ and $180^{\circ}$. The BRDF doesn't change at small scatter zenith angles, while at larger scatter zenith angles the BRDF increases with the wavelength. The detector scan plane is parallel to the weft threads at scatter azimuth angles of $0^{\circ}$ and $180^{\circ}$ and perpendicular to them at a scatter azimuth angle of $90^{\circ}$. The BRDF dependence on the weft threads is best addressed when the BRDF at scatter azimuth angles of $0^{\circ}$ and $90^{\circ}$ are compared. For this comparison, the weft threads influence is presented in Table 6 for sample 1 at wavelengths of 485,550 , and $633 \mathrm{~nm}$. Although in this section discusses the normal incidence related data only, the table includes the non-normal incidence data as well. The BRDF obtained at non-normal incidence is addressed in the respective section. The BRDF measured at a scatter azimuth of $90^{\circ}$ is used as a reference. Since the BRDF at $0^{\circ}$ is always higher than at $90^{\circ}$, the data in the Table are negative. The difference at normal incidence could be as high as $-7.99 \%$ at $60^{\circ}$ scatter zenith at $485 \mathrm{~nm}$ and as low as $-1.17 \%$ at $10^{\circ}$ scatter zenith at $633 \mathrm{~nm}$. The difference in BRDF at $0^{\circ}$ vs $90^{\circ}$ increases with scatter zenith angle for all wavelengths at normal incidence. It is also higher at shorter wavelengths. Although not presented, the results of samples 2, 3 and 4 
show the same characteristics. The variation in BRDF is due to the warp bumps obscuring effect translated into the data at normal incidence.

The BRDF of the tarp samples at non-normal incident angles is different from that at normal incidence. The difference is mainly due to the non-isotropic structure of the sample's surface. The warp bumps (i) partially obscure the detector view and (ii) shadow a portion of the illuminated area of the sample. The two effects reduce the observed BRDF. The influence of these effects on BRDF depends on the incident angle, scatter zenith and azimuth angles, and the orientation of the weft threads relative to the plane of the incident light.

In the process of characterizing the sample BRDF, we fitted the measured points using a standard polynomial procedure, similar to what we used at normal incidence. The coefficients of the polynomial regression are given in Tables 2 and 3 according to the wavelength and measurement geometry for $10^{\circ}$ and $30^{\circ}$ incident angles. The polynomial regression can be used for deriving BRDF data at random scatter zenith angles at fixed scatter azimuth angles at 485 $\mathrm{nm}$. The fitting procedure was applied for scatter zenith angles from $0^{\circ}$ to $60^{\circ}$ except at the $180^{\circ}$ scatter azimuth where it was applied from $15^{\circ}$ to $60^{\circ}$ due to the detector obscuration at $10^{\circ}$ scatter zenith. Two polynomials were used to model the BRDF at the $30^{\circ}$ incident angle geometry, one for $0^{\circ}$ to $25^{\circ}$ scatter zenith and one for $35^{\circ}$ to $60^{\circ}$ scatter zenith as the detector is obscured at the $30^{\circ}$ scatter zenith angle.

BRDF versus scatter zenith angle. BRDF data was acquired at $10^{\circ}$ and $30^{\circ}$ incident angles for $0^{\circ}, 45^{\circ}, 90^{\circ}, 135^{\circ}$ and $180^{\circ}$ scatter azimuth positions on all samples. The scatter zenith angles vary from $0^{\circ}$ to $60^{\circ}$ in $5^{\circ}$ steps. The BRDF of sample 1 at $485 \mathrm{~nm}$ is presented in Figs.9 and 10 for $10^{\circ}$ and $30^{\circ}$ incident angles, respectively. The BRDF of samples 2, 3 and 4 as well as the measurements at 550,633 and $800 \mathrm{~nm}$ are not presented as they show the same scattering tendencies. Two types of data are presented in the figures - the fitted BRDF curve and the measured points. The fitted curves are plotted from the polynomial coefficients as given in Table 2 for $10^{\circ}$ and Table 3 for $30^{\circ}$ incident angles. The BRDF at $10^{\circ}$ incidence angle was seen to 
decrease to a different extent with increasing scatter zenith angle for $0^{\circ}, 45^{\circ}$, and $90^{\circ}$ scatter azimuth independently of wavelength for all samples. The BRDF data at $180^{\circ}$ scatter azimuth follows the tendency as discussed for the case of normal incidence. The BRDF in the principal plane is higher at scatter zenith angles closest to the angle of incidence. The BRDF at $135^{\circ}$ scatter azimuth follows the same pattern although the BRDF values are lower than those measured at $180^{\circ}$ scatter azimuth. Fig. 11 shows the BRDF at $30^{\circ}$ incident angle and $485 \mathrm{~nm}$ for $0^{\circ}, 45^{\circ}, 90^{\circ}, 135^{\circ}$ and $180^{\circ}$ scatter azimuth. The BRDF follows the same pattern as at the $10^{\circ}$ incident angle. However the BRDF at $180^{\circ}$ scatter azimuth is significantly higher than the BRDF at other scatter azimuth angles and at smaller scatter zenith angles.

The BRDF dependence on the weft threads is best represented by comparing the BRDF at scatter azimuth angles of $0^{\circ}$ and $90^{\circ}$. The difference in BRDF of sample 1 at a scatter azimuth $0^{\circ}$ vs. the BRDF at a scatter azimuth of $90^{\circ}$ is presented in Table 6 at 485, 550,633, $800 \mathrm{~nm}$, summarizing data for both normal and non-normal incidence. The BRDF measured at a scatter azimuth of $90^{\circ}$ is used as a reference. Since the BRDF at scatter azimuth $0^{\circ}$ is always higher than at scatter azimuth $90^{\circ}$, the data in the Table are negative. The difference at $30^{\circ}$ incident angle could be as high as $-12 \%$ at $60^{\circ}$ scatter zenith and as low as $0 \%$ at $0^{\circ}$ scatter zenith both at 485 $\mathrm{nm}$.

Backward and forward scatter. The BRDF at non-normal incidence provides information on the forward and backward scattering properties of the tarps. We are considering the BRDF to be in forward direction when the detector is positioned opposite the light source. The backward scatter takes place when the detector is positioned back toward the light source. The typical BRDF has a maximum at the retroscatter position occurring when the detector is aligned on the same line with the source and the sample. The cause of the maximum BRDF is the hiding of shadows and obscuration effects at this geometry. The forward and backward scatters are compared by simply looking at the difference of the BRDF at scatter zenith angles symmetric to the source zenith position. Tarps 1, 2, 3, called "white" tarps hereafter exhibit well-pronounced 
backward scattering, better expressed at larger incident angles for both $10^{\circ}$ and $30^{\circ}$ incident angles as shown for tarp 1 in Fig.11. The "white" tarps' backward scattering is better pronounced at shorter wavelengths. However the scattering of tarp 4, the "black" tarp, is quite different. This sample has well pronounced forward scattering properties as shown in Fig.12, especially at $30^{\circ}$ incident angle. The forward scattering is also apparent for scatter zenith greater than $25^{\circ}$ angles and at $10^{\circ}$ incidence angle.

It is not unusual for black materials to have different scatter distributions than white materials. The tarps used in remote sensing calibrations are generally treated with a pigment of titanium dioxide and carbon black and coated with a silicone pigment. The carbon black exhibits strong forward scattering properties ${ }^{18}$. We believe the forward scattering properties demonstrated by the black sample are due to the carbon black used at the manufacturing process and its polarization properties.

BRDF spectral dependence. The BRDF spectral dependence based on data measured at wavelengths of 485, 550, 633 and $800 \mathrm{~nm}$ is shown in Fig.13. The BRDF of tarp 1 at incident angle $30^{\circ}$, scatter azimuth angles of $0^{\circ}$ and $180^{\circ}$ is presented for scatter zenith angles from $0^{\circ}$ to $60^{\circ}$, in $10^{\circ}$ steps. The BRDF spectral dependence at non-normal incidence follows the same pattern as at normal incidence, that is, the BRDF increases with the wavelength. All the samples were found to have the same spectral dependence, including the black sample. However, the BRDF data of the black sample in the visible $(485,550,633 \mathrm{~nm})$ was very similar, only the BRDF at $800 \mathrm{~nm}$ being higher. This makes the black sample BRDF properties spectrally indifferent in the visible range.

$8^{o}$ directional/hemispherical measurement. The $8^{\circ}$ directional/hemispherical reflectance of all the tarp samples was measured with a HeNe laser at $632.8 \mathrm{~nm}$. The only hardware difference from the experimental setup described in the previous section is the use of an $8^{\circ}$ directional/hemispherical integrating sphere mounted above the scatterometer sample stage. The silicon photodiode detector was fixed to one port of the sphere. The relationship between sample 
reflectance and detector signal can be parameterized using Spectralon samples of known reflectance. We chose a $3^{\text {rd }}$ order polynomial for this parameterization. The coefficients of the polynomial were calculated by fitting the receiver power measured with a set of 7 gray Spectralon standard targets of nominal reflectance $5 \%, 10 \%, 20 \%, 40 \%, 60 \%, 80 \%$ and $99 \%$ with known $8^{\circ}$ directional/hemispherical reflectance. The measured $8^{\circ}$ directional/hemispherical reflectance data for each sample are given in Table 7.

\section{CONCLUSIONS}

The BRDF results of four radiometric tarp samples calibrated at NASA's GSFC Diffuse Calibration Facility show a strong dependence on the weave orientation relative to the measurement geometry. The experimental data shows that the weft and warp threads orientation has a clear effect on BRDF for both normal and non-normal incident angles. Non-normal incident angles introduce an additional dependence of BRDF on weave orientation. BRDF differences vary and can approach $12 \%$ for non-normal incidence and $8 \%$ for normal incident angle. There is also a spectral dependence on BRDF, which is apparent at higher scatter zenith angles over the spectral range from 485 to $800 \mathrm{~nm}$ for both normal and non-normal incidence. The fitted BRDF values show a very small discrepancy from the measured ones in both normal and non-normal incident angles. The highest RMSE was calculated to be $2.32 \times 10^{-4} \mathrm{sr}^{-1}$. The provided polynomial coefficients can be used for calculating the BRDF at random scatter zenith angles. The forward and backward scatter properties of the tarps were also studied. The backward scatter is well pronounced for the "white" samples - tarps 1, 2 and 3. Tarp 4, the "black" sample, has well pronounced forward scatter. The forward scatter is most likely induced by carbon black used in the manufacturing of the darkest sample and its scatter polarization properties. The $8^{\circ}$ directional/hemispherical reflectance data complements the BRDF measurements. 
The current study provides the remote sensing community with important high accuracy BRDF calibration data of radiometric tarps used in the vicarious calibrations of satellite instruments. The BRDF data obtained from these studies is important for future NASA SSC vicarious calibrations through analysis of the BRDF dependence on weft, warp threads orientation. The BRDF characterization methodologies of tarp samples as shown in this paper can be successfully extended to other structured surface fabric samples.

\section{ACKNOWLEDGEMENTS}

The authors would like to thank, among others, Mary Pagnutti and Bob Ryan of NASA's Stennis Space Center for making the tarp samples available to us for measurements, Gil Smith of Science Systems and Applications, Inc. for the critical reading, and to Michael King of NASA's GSFC and Charles Gatebe of University of Maryland Baltimore County for the helpful discussions and recommendations.

This work was supported by the National Aeronautics and Space Administration under Agreement No. NAS5-02041 issued through the Science Mission Directorate. 


\section{REFERENCES}

1. J.J. Butler, B.C. Johnson, R.A. Barnes, "The calibration and characterization of Earth remote sensing and environmental monitoring instruments", in Optical Radiometry, Ed. A.C. Parr, R.U. Datla, J.L. Gardner, Academic Press, New York (2005).

2. G.B. Courreges-Lacoste, J.G. Schaarsberg, R. Sprik, S. Delward, "Modeling of Spectralon diffusers for radiometric calibration in remote sensing", Opt. Eng., 42, 3600-3607 (2003).

3. C.J. Bruegge, A.E. Stiegman, R.A. Rainen and A.W. Springsteen, "Use of Spectralon as a diffuse reflectance standard for in-flight calibration of earth-orbiting sensors", Opt. Eng., 32, 805-814 (1993).

4. C.K. Gatebe, M.D. King, S. Platnick, G.T. Arnold, E.F. Vermote, B. Schmid, “Airborne spectral measurements of surface-atmosphere anisotropy for several surfaces and ecosystems over southern Africa", J. Geophysical Research, 108, D13, 25-1-16 (2003).

5. C.K. Gatebe, M.D. King, A.I. Lyapustin, G.T. Arnold, J. Redemann, “Airborne spectral measurements of ocean directional reflectance", J. Atmospheric Sciences, 62, 1072-1092 (2005).

6. M.D. King, "Directional and spectral reflectance of the Kuwait oil-fire smoke", $J$. Geophysical Research, 97, D13, 14545-14549 (1992).

7. S. Blonski, M. Pagnutti, R.E. Ryan, V. Zanoni, "In-flight edge response measurements for high-spatial-resolution remote sensing systems", Proc. SPIE, 4814, 317-326 (2002). 
8. M. Pagnutti, R. Ryan, M. Kelly, K. Holekamp, V. Zanoni, K Thome, S. Schiller, "Radiometric characterization of IKONOS multispectral imagery" Remote Sensing of Environment, 88, 53-68 (2003).

9. J.R. Proctor and P.Y. Barnes, "NIST High accuracy reference reflectometer spectrophotometer", J. Res. Nat. Inst. Stand. Technol., 101, 619-627 (1996).

10. F.E. Nicodemus, J.C. Richmond, J.J. Hsia, I.W. Ginsburg, and T. Limperis, "Geometrical considerations and nomenclature for reflectance", National Bureau of Standards, NBS monograph 160, Oct. 1977.

11. J.C. Stover, "Optical scattering: measurement and analysis", SPIE Press, Bellingham, Washington, 1995.

12. B.N. Taylor and C. E. Kuyatt, "A Guidelines for Evaluating and Expressing the Uncertainty of NIST Measurement Results", NIST Technical Note 1297, U.S. Department of Commerce, National Institute of Standards and Technology, Sep. 1997.

13. T.F. Schiff, M.W. Knighton, D.J. Wilson, F.M. Cady, J.C. Stover, and J.J. Butler, "A Design Review of a High Accuracy UV to Near Infrared Scatterometer", Proc. SPIE, 1995, 121 130 (1993).

14. F.M. Cady, D.R. Bjork, J. Rifkin, J. C. Stover, "BRDF error analysis", Proc. SPIE, 1165, 154-164 (1989).

15. E.A. Early, P.Y. Barnes, B.C. Johnson, J.J. Butler, C.J. Bruegge, S.F. Biggar, P.S. Spyak and M.M. Pavlov, "Bidirectional Reflectance Round-Robin in Support of the Earth Observing System Program”, J. Atmospheric and Oceanic Techn, 17, 1077-1091 (2000).

16. G.T. Georgiev, J.J. Butler: "Long-term calibration monitoring of spectralon diffusers BRDF in the air-ultraviolet", Applied Optics, 2007, vol.46, No.32, pp.7892-7899. 
17. G.T. Georgiev, J.J. Butler, "Bidirectional reflectance distribution function and directional/hemispherical reflectance of Martian regolith simulant”, Optical Engineering, 44, $036202(2005)$.

18. G.T. Georgiev, C.K. Gatebe, J.J. Butler, M.D. King, "Comparison between laboratory and airborne BRDF measurements for remote sensing”, Proc. SPIE Earth Observing Systems XI, 6296, 629603, 2006.

19. S. Smith, J.C. Fleming, "BRDF measurements of a new IR black coating with lower reflectance”, Proc. SPIE, 3426, 333-343 (1998).

Table 1: Polynomial coefficients of the samples at normal incidence, $485 \mathrm{~nm}$

\begin{tabular}{l}
\hline Coefficients \\
Sample 1 \\
\begin{tabular}{|c|c|c|c|c|c|}
\hline \multicolumn{5}{|c|}{ Scatter azimuth } \\
\hline & 0 & 45 & 90 & 135 & 180 \\
\hline A & 0.20825 & 0.20184 & 0.20172 & 0.20113 & 0.20614 \\
\hline B & $-3.69548 \times 10^{-3}$ & $-2.52895 \times 10^{-3}$ & $-2.95644 \times 10^{-3}$ & $-2.80741 \times 10^{-3}$ & $-3.32718 \times 10^{-3}$ \\
\hline C & $1.63404 \times 10^{-4}$ & $6.91565 \times 10^{-5}$ & $9.51081 \times 10^{-5}$ & $9.11978 \times 10^{-5}$ & $1.36292 \times 10^{-4}$ \\
\hline D & $-4.18955 \times 10^{-6}$ & $-9.95396 \times 10^{-7}$ & $-2.02885 \times 10^{-6}$ & $-1.99668 \times 10^{-6}$ & $-3.43818 \times 10^{-6}$ \\
\hline E & $5.37358 \times 10^{-8}$ & $3.71795 \times 10^{-9}$ & $2.31818 \times 10^{-8}$ & $2.33217 \times 10^{-8}$ & $4.48814 \times 10^{-8}$ \\
\hline F & $-2.68175 \times 10^{-10}$ & $2.5641 \times 10^{-11}$ & $-1.07692 \times 10^{-10}$ & $-1.07692 \times 10^{-10}$ & $-2.31976 \times 10^{-10}$ \\
\hline
\end{tabular}
\end{tabular}

Sample 2

\begin{tabular}{|l|l|l|l|l|l|}
\hline A & 0.16405 & 0.16329 & 0.15781 & 0.16607 & 0.16417 \\
\hline B & $-3.52082 \times 10^{-3}$ & $-3.80467 \times 10^{-3}$ & $-2.95437 \times 10^{-3}$ & $-4.54988 \times 10^{-3}$ & $-3.73089 \times 10^{-3}$ \\
\hline C & $1.41832 \times 10^{-4}$ & $1.67633 \times 10^{-4}$ & $9.67351 \times 10^{-5}$ & $2.19402 \times 10^{-4}$ & $1.62164 \times 10^{-4}$ \\
\hline D & $-3.40202 \times 10^{-6}$ & $-4.48153 \times 10^{-6}$ & $-2.16148 \times 10^{-6}$ & $-6.30198 \times 10^{-6}$ & $-4.10819 \times 10^{-6}$ \\
\hline E & $4.10483 \times 10^{-8}$ & $6.01981 \times 10^{-8}$ & $2.6352 \times 10^{-8}$ & $9.06993 \times 10^{-8}$ & $5.18333 \times 10^{-8}$ \\
\hline F & $-1.91855 \times 10^{-10}$ & $-3.12821 \times 10^{-10}$ & $-1.28205 \times 10^{-10}$ & $-5.02564 \times 10^{-10}$ & $-2.5098 \times 10^{-10}$ \\
\hline
\end{tabular}

Sample 3

\begin{tabular}{|l|l|l|l|l|l|}
\hline A & 0.10311 & 0.0977 & 0.0995 & 0.0974 & 0.10218 \\
\hline B & $-2.38324 \times 10^{-3}$ & $-1.55687 \times 10^{-3}$ & $-2.07108 \times 10^{-3}$ & $-1.61648 \times 10^{-3}$ & $-2.29836 \times 10^{-3}$ \\
\hline C & $9.63805 \times 10^{-5}$ & $4.05597 \times 10^{-5}$ & $6.94231 \times 10^{-5}$ & $4.15026 \times 10^{-5}$ & $9.2933 \times 10^{-5}$ \\
\hline D & $-2.25495 \times 10^{-6}$ & $-6.38753 \times 10^{-7}$ & $-1.5035 \times 10^{-6}$ & $-6.49476 \times 10^{-7}$ & $-2.1905 \times 10^{-6}$ \\
\hline E & $2.69601 \times 10^{-8}$ & $5.20979 \times 10^{-9}$ & $1.7669 \times 10^{-8}$ & $5.34965 \times 10^{-9}$ & $2.65967 \times 10^{-8}$ \\
\hline F & $-1.26998 \times 10^{-10}$ & $-1.53846 \times 10^{-11}$ & $-8.20513 \times 10^{-11}$ & $-1.53846 \times 10^{-11}$ & $-1.28205 \times 10^{-10}$ \\
\hline
\end{tabular}




\section{Sample 4}

\begin{tabular}{|c|c|c|c|c|c|}
\hline A & 0.01092 & 0.01005 & $8.46818 \times 10^{-3}$ & $9.39545 \times 10^{-3}$ & 0.01039 \\
\hline B & $-2.41551 \times 10^{-4}$ & $-1.29779 \times 10^{-5}$ & $2.42244 \times 10^{-4}$ & $5.50635 \times 10^{-5}$ & $-1.08233 \times 10^{-4}$ \\
\hline C & $1.3284 \times 10^{-5}$ & $-7.4528 \times 10^{-6}$ & $-2.24962 \times 10^{-5}$ & $-9.82197 \times 10^{-6}$ & $1.75257 \times 10^{-6}$ \\
\hline D & $-3.65361 \times 10^{-7}$ & $3.98485 \times 10^{-7}$ & $7.69172 \times 10^{-7}$ & $3.85839 \times 10^{-7}$ & $5.11758 \times 10^{-8}$ \\
\hline E & $4.59345 \times 10^{-9}$ & $-7.71562 \times 10^{-9}$ & $-1.16667 \times 10^{-8}$ & $-6.18881 \times 10^{-9}$ & $-2.0074 \times 10^{-9}$ \\
\hline F & $-2.14178 \times 10^{-11}$ & $5.12821 \times 10^{-11}$ & $6.66667 \times 10^{-11}$ & $3.58974 \times 10^{-11}$ & $1.71946 \times 10^{-11}$ \\
\hline
\end{tabular}

Table 2: Polynomial coefficients of the samples at 10 deg incidence, $485 \mathrm{~nm}$

\begin{tabular}{|c|c|}
\hline Coefficients & Scatter azimuth \\
\hline
\end{tabular}

\section{Sample 1}

\begin{tabular}{|l|l|l|l|l|l|}
\hline & 0 & \multicolumn{1}{|c|}{45} & \multicolumn{1}{c|}{90} & \multicolumn{1}{c|}{135} & 180 \\
\hline A & 0.18296 & 0.18354 & 0.18312 & 0.18365 & 0.28976 \\
\hline B & $-1.20021 \times 10^{-3}$ & $-9.69627 \times 10^{-4}$ & $1.11966 \times 10^{-4}$ & $1.74708 \times 10^{-3}$ & -0.01248 \\
\hline C & $2.15828 \times 10^{-5}$ & $2.51107 \times 10^{-6}$ & $-9.95822 \times 10^{-5}$ & $-2.2565 \times 10^{-4}$ & $5.94409 \times 10^{-4}$ \\
\hline D & $-1.37951 \times 10^{-7}$ & $3.74383 \times 10^{-7}$ & $3.87859 \times 10^{-6}$ & $7.98647 \times 10^{-6}$ & $-1.5002 \times 10^{-5}$ \\
\hline E & $-1.79899 \times 10^{-9}$ & $-8.2586 \times 10^{-9}$ & $-6.19032 \times 10^{-8}$ & $-1.23052 \times 10^{-7}$ & $1.88765 \times 10^{-7}$ \\
\hline F & $2.32278 \times 10^{-11}$ & $5.3997 \times 10^{-11}$ & $3.59276 \times 10^{-10}$ & $7.01357 \times 10^{-10}$ & $-9.31282 \times 10^{-10}$ \\
\hline
\end{tabular}

Sample 2

\begin{tabular}{|l|l|l|l|l|l|}
\hline A & 0.13859 & 0.13867 & 0.13873 & 0.13875 & 0.24535 \\
\hline B & $-1.36321 \times 10^{-3}$ & $-9.63364 \times 10^{-4}$ & $-2.23778 \times 10^{-4}$ & $1.74996 \times 10^{-3}$ & -0.01204 \\
\hline C & $3.90652 \times 10^{-5}$ & $-2.29028 \times 10^{-6}$ & $-6.973 \times 10^{-5}$ & $-2.19264 \times 10^{-4}$ & $5.41148 \times 10^{-4}$ \\
\hline D & $-9.58556 \times 10^{-7}$ & $5.30793 \times 10^{-7}$ & $2.82008 \times 10^{-6}$ & $7.65497 \times 10^{-6}$ & $-1.26653 \times 10^{-5}$ \\
\hline E & $1.33374 \times 10^{-8}$ & $-1.02153 \times 10^{-8}$ & $-4.4891 \times 10^{-8}$ & $-1.16757 \times 10^{-7}$ & $1.46154 \times 10^{-7}$ \\
\hline F & $-7.30015 \times 10^{-11}$ & $6.36501 \times 10^{-11}$ & $2.6003 \times 10^{-10}$ & $6.61538 \times 10^{-10}$ & $-6.5641 \times 10^{-10}$ \\
\hline
\end{tabular}

\section{Sample 3}

\begin{tabular}{|l|l|l|l|l|l|}
\hline A & 0.08608 & 0.08612 & 0.08621 & 0.08609 & 0.14903 \\
\hline B & $-9.23378 \times 10^{-4}$ & $-6.93915 \times 10^{-4}$ & $-1.81439 \times 10^{-4}$ & $1.09972 \times 10^{-3}$ & $-6.82608 \times 10^{-3}$ \\
\hline C & $2.54172 \times 10^{-5}$ & $1.76028 \times 10^{-6}$ & $-4.5006 \times 10^{-5}$ & $-1.40507 \times 10^{-4}$ & $2.94509 \times 10^{-4}$ \\
\hline D & $-4.75932 \times 10^{-7}$ & $3.40265 \times 10^{-7}$ & $1.89407 \times 10^{-6}$ & $4.89841 \times 10^{-6}$ & $-6.80331 \times 10^{-6}$ \\
\hline E & $5.38599 \times 10^{-9}$ & $-7.49074 \times 10^{-9}$ & $-3.05882 \times 10^{-8}$ & $-7.39696 \times 10^{-8}$ & $7.92075 \times 10^{-8}$ \\
\hline F & $-2.62443 \times 10^{-11}$ & $5.12821 \times 10^{-11}$ & $1.79487 \times 10^{-10}$ & $4.14178 \times 10^{-10}$ & $-3.65128 \times 10^{-10}$ \\
\hline
\end{tabular}




\section{Sample 4}

\begin{tabular}{|l|l|l|l|l|l|}
\hline A & $9.48484 \times 10^{-3}$ & $9.48213 \times 10^{-3}$ & $9.51946 \times 10^{-3}$ & $9.50271 \times 10^{-3}$ & 0.01512 \\
\hline B & $-1.9931 \times 10^{-5}$ & $-1.30863 \times 10^{-5}$ & $-2.16218 \times 10^{-5}$ & $6.22498 \times 10^{-5}$ & $-7.10272 \times 10^{-4}$ \\
\hline C & $-2.58155 \times 10^{-6}$ & $-3.54336 \times 10^{-6}$ & $-2.97816 \times 10^{-6}$ & $-1.06857 \times 10^{-5}$ & $3.47308 \times 10^{-5}$ \\
\hline D & $1.91907 \times 10^{-7}$ & $2.05142 \times 10^{-7}$ & $1.17267 \times 10^{-7}$ & $4.06767 \times 10^{-7}$ & $-8.93986 \times 10^{-7}$ \\
\hline E & $-4.0436 \times 10^{-9}$ & $-3.96682 \times 10^{-9}$ & $-1.6221 \times 10^{-9}$ & $-6.59948 \times 10^{-9}$ & $1.14219 \times 10^{-8}$ \\
\hline F & $2.7451 \times 10^{-11}$ & $2.6546 \times 10^{-11}$ & $8.74811 \times 10^{-12}$ & $3.92157 \times 10^{-11}$ & $-5.74359 \times 10^{-11}$ \\
\hline
\end{tabular}

Table 3: Polynomial coefficients of the samples at 30 deg incidence, $485 \mathrm{~nm}$

\begin{tabular}{|c|c|c|c|c|c|}
\hline Coefficients & \multicolumn{5}{|c|}{ Scatter azimuth, deg } \\
\hline & $0^{\circ}$ & $45^{\circ}$ & $90^{\circ}$ & $135^{\circ}$ & \multicolumn{2}{c|}{$180^{\circ}$} \\
\hline
\end{tabular}

Sample 1

\begin{tabular}{|c|c|c|c|c|c|c|}
\hline A & 0.16484 & 0.16479 & 0.16483 & 0.16467 & 0.1648 & 2.6852 \\
\hline B & $-7.09245 \times 10^{-4}$ & $-4.62207 \times 10^{-4}$ & $2.03307 \times 10^{-4}$ & $7.44778 \times 10^{-4}$ & $6.15333 \times 10^{-4}$ & -0.24781 \\
\hline C & $1.45158 \times 10^{-5}$ & $1.4295 \times 10^{-5}$ & $2.61323 \times 10^{-5}$ & $-7.18017 \times 10^{-6}$ & $5.76667 \times 10^{-5}$ & $9.959 \times 10^{-3}$ \\
\hline D & $1.93459 \times 10^{-7}$ & $-6.41475 \times 10^{-7}$ & $5.84595 \times 10^{-7}$ & $7.11261 \times 10^{-7}$ & $-3.83333 \times 10^{-6}$ & $-2.01367 \times 10^{-4}$ \\
\hline E & $7.59633 \times 10^{-10}$ & $1.23776 \times 10^{-8}$ & $-6.8696 \times 10^{-9}$ & $1.65611 \times 10^{-8}$ & $9.33333 \times 10^{-8}$ & $2.04 \times 10^{-6}$ \\
\hline F & $8.44646 \times 10^{-12}$ & $-7.93364 \times 10^{-11}$ & $3.68024 \times 10^{-11}$ & $-1.06486 \times 10^{-10}$ & $8 \times 10^{-10}$ & $-8.26667 \times 10^{-9}$ \\
\hline
\end{tabular}

Sample 2

\begin{tabular}{|c|c|c|c|c|c|c|}
\hline A & 0.1226 & 0.12245 & 0.12244 & 0.12249 & 0.1225 & 2.5561 \\
\hline B & $-7.37016 \times 10^{-4}$ & $-3.02171 \times 10^{-4}$ & $1.98946 \times 10^{-4}$ & $6.47981 \times 10^{-4}$ & $3.90333 \times 10^{-4}$ & -0.23511 \\
\hline C & $1.38939 \times 10^{-5}$ & $-1.48631 \times 10^{-5}$ & $-3.30949 \times 10^{-5}$ & $-4.34286 \times 10^{-6}$ & $1.34167 \times 10^{-4}$ & $9.2725 \times 10^{-3}$ \\
\hline D & $-1.89946 \times 10^{-7}$ & $6.02033 \times 10^{-7}$ & $8.51464 \times 10^{-7}$ & $-7.23382 \times 10^{-7}$ & $-1.30333 \times 10^{-5}$ & $-1.837 \times 10^{-4}$ \\
\hline E & $7.65117 \times 10^{-10}$ & $-8.85918 \times 10^{-9}$ & $-1.02797 \times 10^{-8}$ & $1.55492 \times 10^{-8}$ & $5.53333 \times 10^{-7}$ & $1.82 \times 10^{-6}$ \\
\hline F & $8.74811 \times 10^{-12}$ & $4.97738 \times 10^{-11}$ & $5.33937 \times 10^{-11}$ & $-9.2006 \times 10^{-11}$ & $-7.2 \times 10^{-9}$ & $-7.2 \times 10^{-9}$ \\
\hline
\end{tabular}

\section{Sample 3}

\begin{tabular}{|c|c|c|c|c|c|c|}
\hline A & 0.07348 & 0.0735 & 0.07351 & 0.0735 & 0.0735 & 1.4191 \\
\hline B & $-4.83623 \times 10^{-4}$ & $-2.90567 \times 10^{-4}$ & $6.10769 \times 10^{-5}$ & $3.87863 \times 10^{-4}$ & $6.41333 \times 10^{-4}$ & -0.12745 \\
\hline C & $8.17157 \times 10^{-6}$ & $-2.45451 \times 10^{-6}$ & $-1.10758 \times 10^{-5}$ & $3.42037 \times 10^{-6}$ & $-4.03333 \times 10^{-5}$ & $4.96017 \times 10^{-3}$ \\
\hline D & $-1.1751 \times 10^{-8}$ & $2.9118 \times 10^{-7}$ & $3.14452 \times 10^{-7}$ & $-3.62923 \times 10^{-7}$ & $6.2 \times 10^{-6}$ & $-9.72 \times 10^{-5}$ \\
\hline E & $-1.3177 \times 10^{-9}$ & $-5.86727 \times 10^{-9}$ & $-3.9627 \times 10^{-9}$ & $4.70725 \times 10^{-9}$ & $-3.06667 \times 10^{-7}$ & $9.53333 \times 10^{-7}$ \\
\hline
\end{tabular}




\begin{tabular}{|c|c|c|c|c|c|c|}
\hline $\mathrm{F}$ & $1.68929 \times 10^{-11}$ & $4.19306 \times 10^{-11}$ & $2.05128 \times 10^{-11}$ & $-1.56863 \times 10^{-11}$ & $5.86667 \times 10^{-9}$ & $-3.73333 \times 10^{-9}$ \\
\hline
\end{tabular}

Sample 4

\begin{tabular}{|c|c|c|c|c|c|c|}
\hline A & $8.88778 \times 10^{-3}$ & $8.90136 \times 10^{-3}$ & $8.90407 \times 10^{-3}$ & $8.88484 \times 10^{-3}$ & $8.9 \times 10^{-3}$ & 0.1738 \\
\hline B & $-8.611 \times 10^{-7}$ & $-2.09143 \times 10^{-5}$ & $4.48396 \times 10^{-6}$ & $-1.71592 \times 10^{-5}$ & $-1.05 \times 10^{-4}$ & -0.01642 \\
\hline C & $1.76189 \times 10^{-8}$ & $5.2077 \times 10^{-7}$ & $-2.16554 \times 10^{-6}$ & $2.1775 \times 10^{-6}$ & $2.75 \times 10^{-5}$ & $6.58667 \times 10^{-4}$ \\
\hline D & $-2.36323 \times 10^{-8}$ & $-2.6704 \times 10^{-7}$ & $9.82723 \times 10^{-8}$ & $-1.52969 \times 10^{-7}$ & $-2.56667 \times 10^{-6}$ & $-1.32667 \times 10^{-5}$ \\
\hline E & $1.28891 \times 10^{-10}$ & $5.53819 \times 10^{-9}$ & $-1.91828 \times 10^{-9}$ & $3.03579 \times 10^{-9}$ & $1 \times 10^{-7}$ & $1.33333 \times 10^{-7}$ \\
\hline F & $3.01659 \times 10^{-12}$ & $-3.65008 \times 10^{-11}$ & $1.44796 \times 10^{-11}$ & $-1.93062 \times 10^{-11}$ & $-1.33333 \times 10^{-9}$ & $-5.33333 \times 10^{-10}$ \\
\hline
\end{tabular}

Table 4: The Root-Mean-Square Error, $\mathrm{sr}^{-1}$, sample 1, $485 \mathrm{~nm}$

\begin{tabular}{|c|c|c|c|c|c|c|}
\hline $\begin{array}{c}\text { Incident } \\
\text { angle, deg }\end{array}$ & \multicolumn{5}{|c|}{ Scatter azimuth, deg } \\
\hline & $0^{0}$ & $45^{0}$ & $90^{0}$ & $135^{\circ}$ & \multicolumn{2}{c|}{$180^{0}$} \\
\hline $0^{0}$ & $2.32 \times 10^{-4}$ & $1.37 \times 10^{-4}$ & $1.20^{-4}$ & $6.87 \times 10^{-5}$ & $8.88 \times 10^{-5}$ & \\
\hline $10^{0}$ & $1.53 \times 10^{-04}$ & $2.06 \times 10^{-04}$ & $1.73 \times 10^{-04}$ & $2.86 \times 10^{-04}$ & $1.60 \times 10^{-04}$ & \\
\hline $30^{\circ}$ & $1.81 \times 10^{-04}$ & $1.71 \times 10^{-04}$ & $1.85 \times 10^{-04}$ & $1.98 \times 10^{-04}$ & $1.22 \times 10^{-13}$ & $6.78 \times 10^{-09}$ \\
\hline
\end{tabular}

Table 5: The BRDF difference from a reference (BRDF at $30^{\circ}$ scatter zenith) measured at corresponding scatter zenith and azimuth angles, at normal incidence, sample 1, $485 \mathrm{~nm}$

\begin{tabular}{|c|c|c|c|c|c|}
\hline \multirow{2}{*}{$\begin{array}{c}\text { Scatter zenith, } \\
\text { deg }\end{array}$} & \multicolumn{5}{|c|}{ Scatter azimuth, deg } \\
\hline & $0^{\circ}$ & $45^{\circ}$ & $90^{\circ}$ & $135^{\circ}$ & $180^{\circ}$ \\
\hline 5 & $13.02 \%$ & & & & $13.35 \%$ \\
\hline 10 & $8.28 \%$ & $9.70 \%$ & $11.17 \%$ & $10.59 \%$ & $9.10 \%$ \\
\hline 15 & $5.66 \%$ & $6.58 \%$ & $7.58 \%$ & $7.25 \%$ & $5.98 \%$ \\
\hline 20 & $3.22 \%$ & $3.85 \%$ & $4.77 \%$ & $4.50 \%$ & $3.53 \%$ \\
\hline 25 & $1.52 \%$ & $2.08 \%$ & $2.44 \%$ & $2.12 \%$ & $1.82 \%$ \\
\hline 30 & $0.00 \%$ & $0.00 \%$ & $0.00 \%$ & $0.00 \%$ & $0.00 \%$ \\
\hline 35 & $-1.51 \%$ & $-1.54 \%$ & $-1.91 \%$ & $-1.90 \%$ & $-1.52 \%$ \\
\hline
\end{tabular}


Laboratory-based bidirectional reflectance distribution functions of radiometric tarps

\begin{tabular}{|l|l|l|l|l|l|}
\hline 40 & $-2.75 \%$ & $-3.13 \%$ & $-3.63 \%$ & $-3.53 \%$ & $-2.77 \%$ \\
\hline 45 & $-3.70 \%$ & $-4.77 \%$ & $-5.34 \%$ & $-5.29 \%$ & $-4.05 \%$ \\
\hline 50 & $-4.99 \%$ & $-6.19 \%$ & $-7.10 \%$ & $-6.68 \%$ & $-5.04 \%$ \\
\hline 55 & $-5.98 \%$ & $-7.57 \%$ & $-8.56 \%$ & $-7.75 \%$ & $-6.04 \%$ \\
\hline 60 & $-6.65 \%$ & $-8.28 \%$ & $-10.06 \%$ & $-8.84 \%$ & $-7.06 \%$ \\
\hline
\end{tabular}

Table 6: BRDF difference at scatter azimuth $0^{\circ}$ vs. $90^{\circ}, \%$, sample $1,90^{\circ}$ is the base 


\begin{tabular}{|c|c|c|c|c|}
\hline Scatter zenith & $485 \mathrm{~nm}$ & $550 \mathrm{~nm}$ & $633 \mathrm{~nm}$ & $800 \mathrm{~nm}$ \\
\hline
\end{tabular}

Normal incidence

\begin{tabular}{|c|c|c|c|c|}
\hline $10^{\circ}$ & $-2.00 \%$ & $-1.74 \%$ & $-1.17 \%$ & $-1.29 \%$ \\
\hline $15^{\circ}$ & $-2.89 \%$ & $-2.32 \%$ & $-2.16 \%$ & $-2.02 \%$ \\
\hline $20^{\circ}$ & $-3.34 \%$ & $-2.82 \%$ & $-2.95 \%$ & $-2.35 \%$ \\
\hline $25^{0}$ & $-4.03 \%$ & $-3.86 \%$ & $-3.37 \%$ & $-2.74 \%$ \\
\hline $30^{\circ}$ & $-4.85 \%$ & $-4.06 \%$ & $-4.04 \%$ & $-3.39 \%$ \\
\hline $35^{\circ}$ & $-5.26 \%$ & $-4.57 \%$ & $-4.70 \%$ & $-3.72 \%$ \\
\hline $40^{\circ}$ & $-5.74 \%$ & $-5.28 \%$ & $-4.88 \%$ & $-4.20 \%$ \\
\hline $45^{\circ}$ & $-6.33 \%$ & $-5.90 \%$ & $-5.42 \%$ & $-4.87 \%$ \\
\hline $50^{\circ}$ & $-6.94 \%$ & $-6.31 \%$ & $-5.70 \%$ & $-4.71 \%$ \\
\hline $55^{0}$ & $-7.37 \%$ & $-6.70 \%$ & $-6.59 \%$ & $-5.05 \%$ \\
\hline $60^{\circ}$ & $-7.99 \%$ & $-7.10 \%$ & $-6.64 \%$ & $-5.40 \%$ \\
\hline
\end{tabular}

$10^{\circ}$ incident angle

\begin{tabular}{|c|c|c|c|c|}
\hline $0^{0}$ & $0.00 \%$ & $0.00 \%$ & $0.14 \%$ & $0.00 \%$ \\
\hline $5^{\circ}$ & $-2.06 \%$ & $-1.69 \%$ & $-1.41 \%$ & $-1.77 \%$ \\
\hline $15^{\circ}$ & $-5.57 \%$ & $-4.97 \%$ & $-4.51 \%$ & $-4.02 \%$ \\
\hline $20^{\circ}$ & $-4.68 \%$ & $-4.01 \%$ & $-3.72 \%$ & $-3.22 \%$ \\
\hline $25^{\circ}$ & $-4.91 \%$ & $-4.13 \%$ & $-3.79 \%$ & $-3.09 \%$ \\
\hline $30^{\circ}$ & $-5.18 \%$ & $-4.19 \%$ & $-4.15 \%$ & $-3.49 \%$ \\
\hline $35^{\circ}$ & $-5.59 \%$ & $-4.90 \%$ & $-4.20 \%$ & $-3.80 \%$ \\
\hline $40^{\circ}$ & $-5.91 \%$ & $-5.12 \%$ & $-4.72 \%$ & $-4.14 \%$ \\
\hline $45^{\circ}$ & $-6.64 \%$ & $-6.24 \%$ & $-5.42 \%$ & $-4.79 \%$ \\
\hline $50^{\circ}$ & $-6.89 \%$ & $-6.10 \%$ & $-5.63 \%$ & $-4.82 \%$ \\
\hline $55^{\circ}$ & $-7.82 \%$ & $-7.01 \%$ & $-6.58 \%$ & $-5.65 \%$ \\
\hline $60^{\circ}$ & $-8.24 \%$ & $-6.89 \%$ & $-6.59 \%$ & $-5.66 \%$ \\
\hline
\end{tabular}

$30^{\circ}$ incident angle

\begin{tabular}{|c|c|c|c|c|}
\hline $0^{\circ}$ & $0.00 \%$ & $0.00 \%$ & $-0.18 \%$ & $-0.15 \%$ \\
\hline $5^{\circ}$ & $-0.03 \%$ & $0.12 \%$ & $0.18 \%$ & $0.15 \%$ \\
\hline $10^{\circ}$ & $-0.94 \%$ & $-0.48 \%$ & $-0.42 \%$ & $-0.70 \%$ \\
\hline $15^{\circ}$ & $-2.63 \%$ & $-1.98 \%$ & $-2.12 \%$ & $-1.76 \%$ \\
\hline $20^{\circ}$ & $-5.75 \%$ & $-4.76 \%$ & $-4.12 \%$ & $-3.98 \%$ \\
\hline $25^{\circ}$ & $-10.51 \%$ & $-8.82 \%$ & $-7.98 \%$ & $-7.01 \%$ \\
\hline $35^{\circ}$ & $-13.46 \%$ & $-11.60 \%$ & $-11.06 \%$ & $-9.71 \%$ \\
\hline $40^{\circ}$ & $-11.76 \%$ & $-9.82 \%$ & $-9.11 \%$ & $-8.26 \%$ \\
\hline $45^{\circ}$ & $-11.56 \%$ & $-9.57 \%$ & $-9.01 \%$ & $-8.46 \%$ \\
\hline $50^{\circ}$ & $-11.34 \%$ & $-9.82 \%$ & $-9.22 \%$ & $-8.63 \%$ \\
\hline $55^{\circ}$ & $-11.42 \%$ & $-10.12 \%$ & $-9.44 \%$ & $-9.15 \%$ \\
\hline $60^{\circ}$ & $-12.04 \%$ & $-11.03 \%$ & $-10.30 \%$ & $-9.80 \%$ \\
\hline
\end{tabular}


Table 7: $8^{0}$ directional/hemispherical reflectance at $632.8 \mathrm{~nm}$

\begin{tabular}{|c|c|}
\hline Sample & Hemispherical Reflectance \\
\hline 1 & $67.34 \%$ \\
\hline 2 & $49.65 \%$ \\
\hline 3 & $31.93 \%$ \\
\hline 4 & $4.35 \%$ \\
\hline
\end{tabular}




\section{FIGURE CAPTIONS:}

Fig.1: BRDF geometry of incident and reflected elementary beams after Nicodemus

Fig.2: NASA - GSFC scatterometer optical setup

Fig.3: NASA - GSFC scatterometer goniometer mechanism

Fig.4: Microscopic image of tarp sample 1, where the warp threads are horizontal and weft threads are vertical

Fig.5: In-plane BRDF of tarps 1 to 4 at normal incidence, $485 \mathrm{~nm}, 0^{\circ}$ and $180^{\circ}$ scatter azimuth angle

Fig.6: BRDF of tarp 1 at normal incidence, at different scatter azimuth angles, $485 \mathrm{~nm}$, fitting and experimental points

Fig.7: Shadowing and obscuration effects of tarps

Fig.8: BRDF of tarp 1 at normal incidence, $485,550,633$, and $800 \mathrm{~nm}, 0^{\circ}$ and $180^{\circ}$ scatter azimuth angle

Fig.9: BRDF of tarp 1 at $10^{\circ}$ incidence at different scatter azimuth angles, $485 \mathrm{~nm}$, fitting and experimental points

Fig.10: BRDF of tarp 1 at $30^{\circ}$ incidence at different scatter azimuth angles, $485 \mathrm{~nm}$, fitting and experimental points

Fig.11: Forward - Backward scatter, tarp 1, 485 and $800 \mathrm{~nm}, 10^{\circ}$ and $30^{\circ}$ incident angle

Fig.12: Forward - Backward scatter, tarp 4, 485 and $800 \mathrm{~nm}, 10^{\circ}$ and $30^{\circ}$ incident angle

Fig.13: Tarp sample 1 at $30^{\circ}$ incidence, $485 \mathrm{~nm}, 550 \mathrm{~nm}, 633 \mathrm{~nm}$, and $800 \mathrm{~nm}$ 


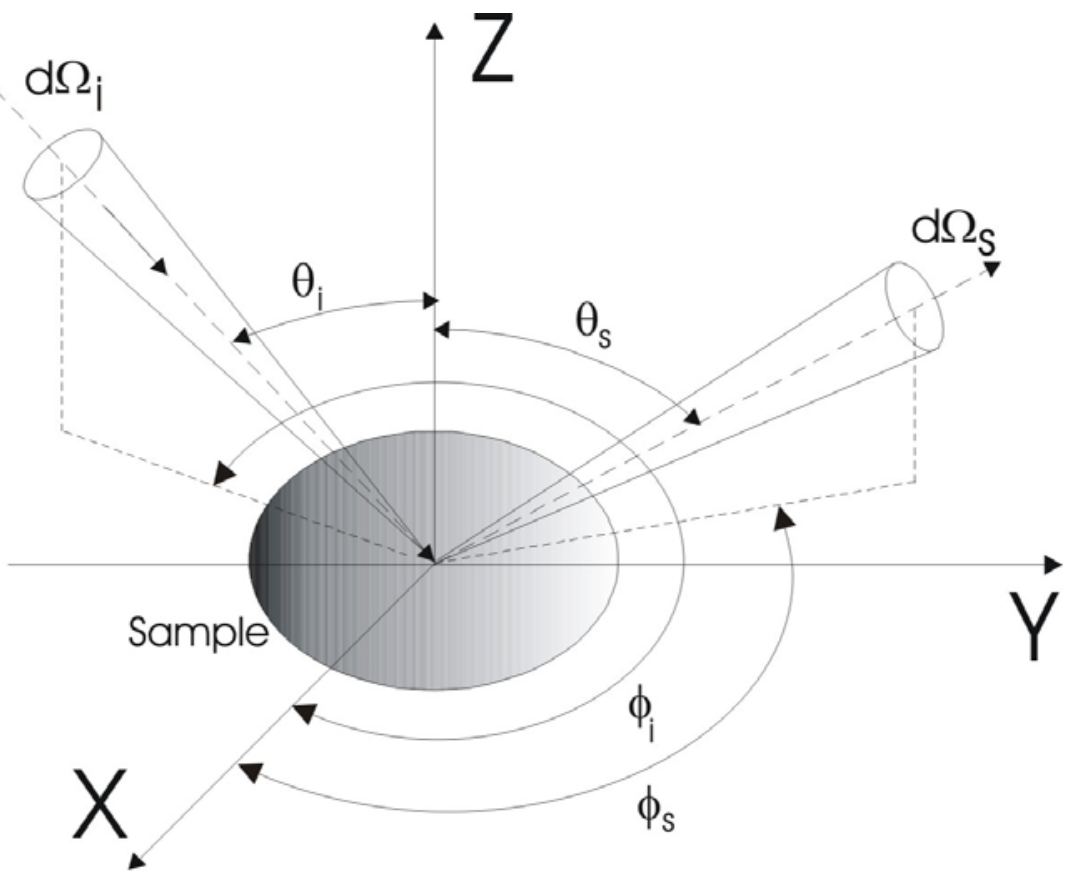

Fig.1: BRDF geometry of incident and reflected elementary beams after Nicodemus 


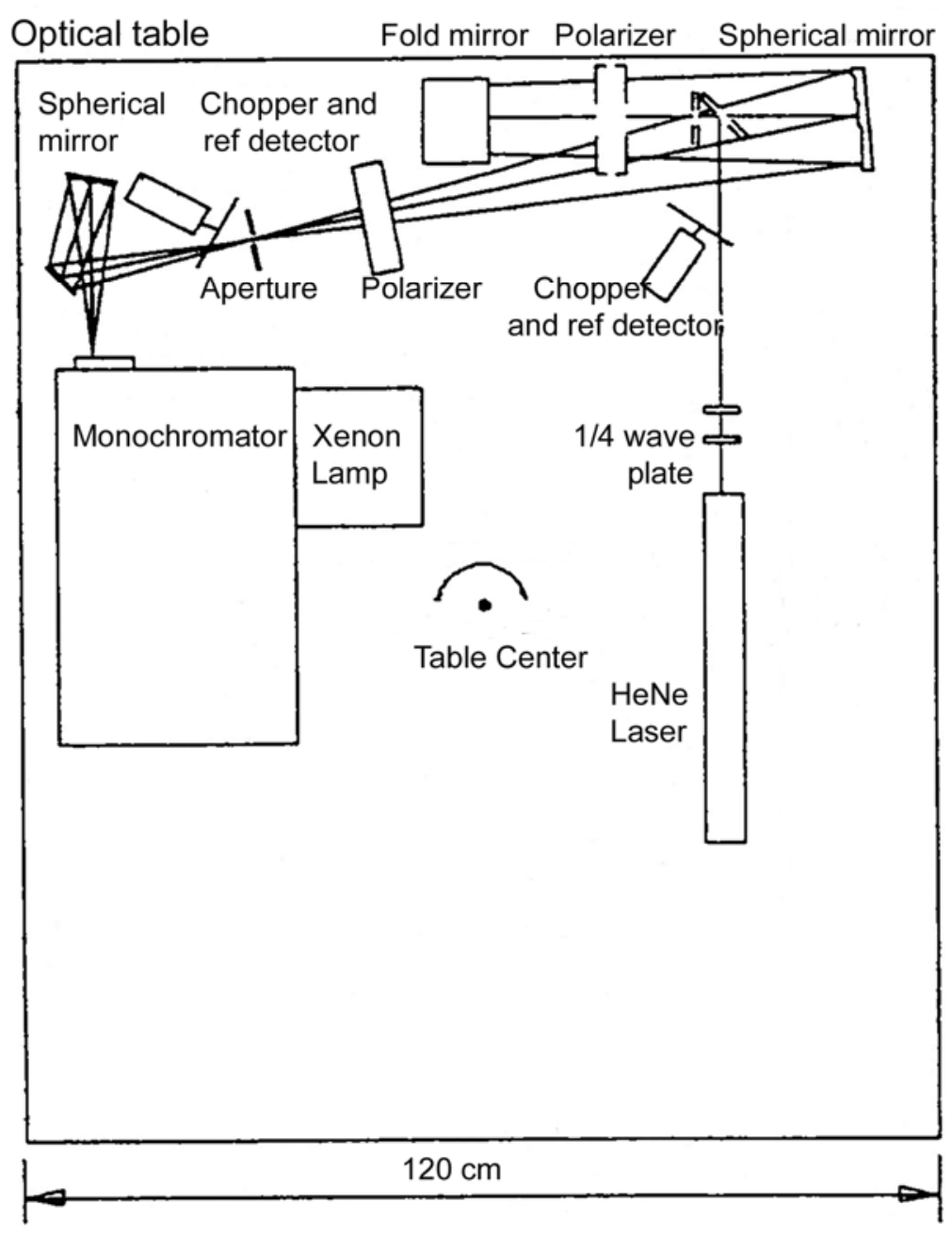

Fig.2: NASA - GSFC scatterometer optical setup 

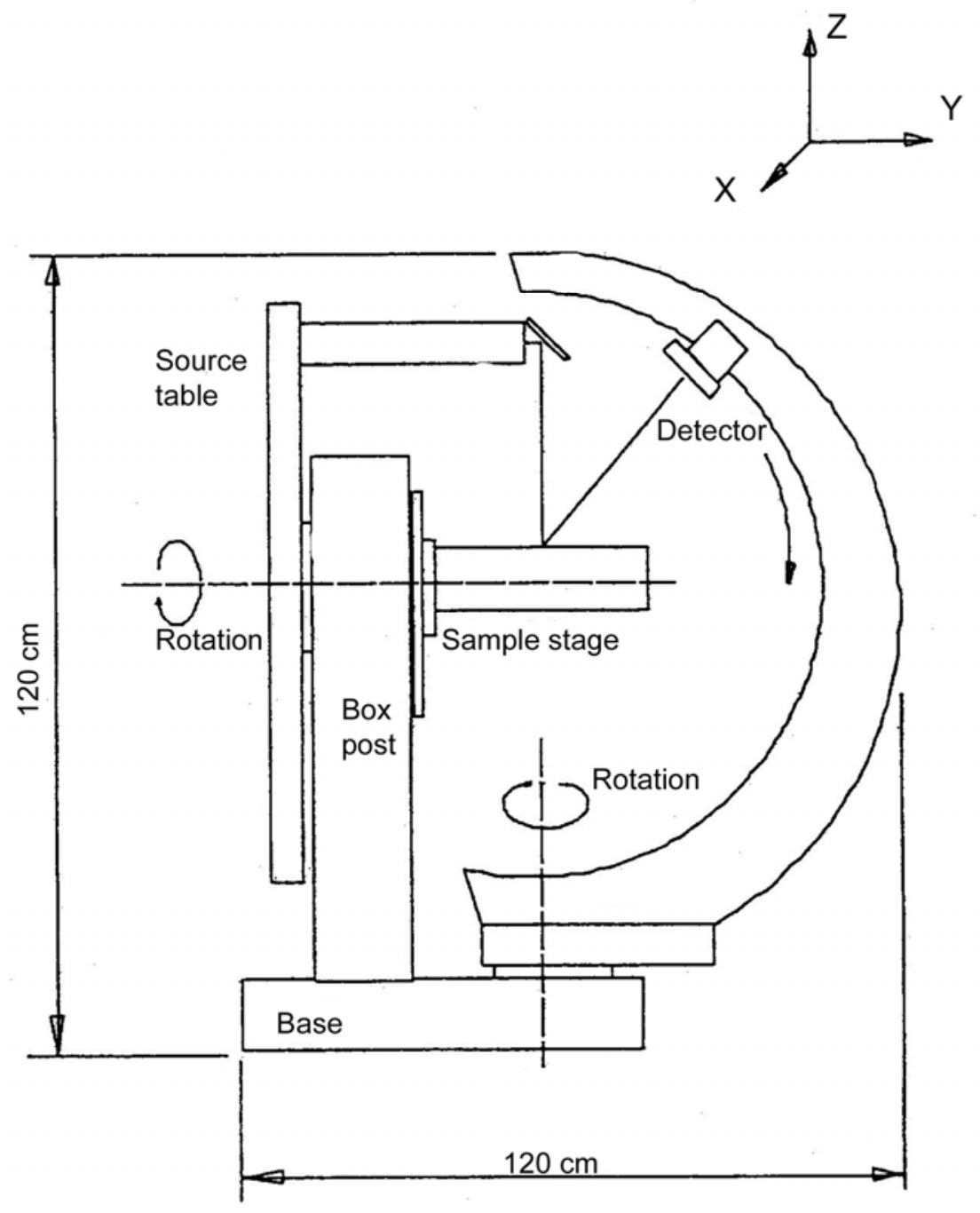

Fig.3: NASA - GSFC scatterometer goniometer mechanism 


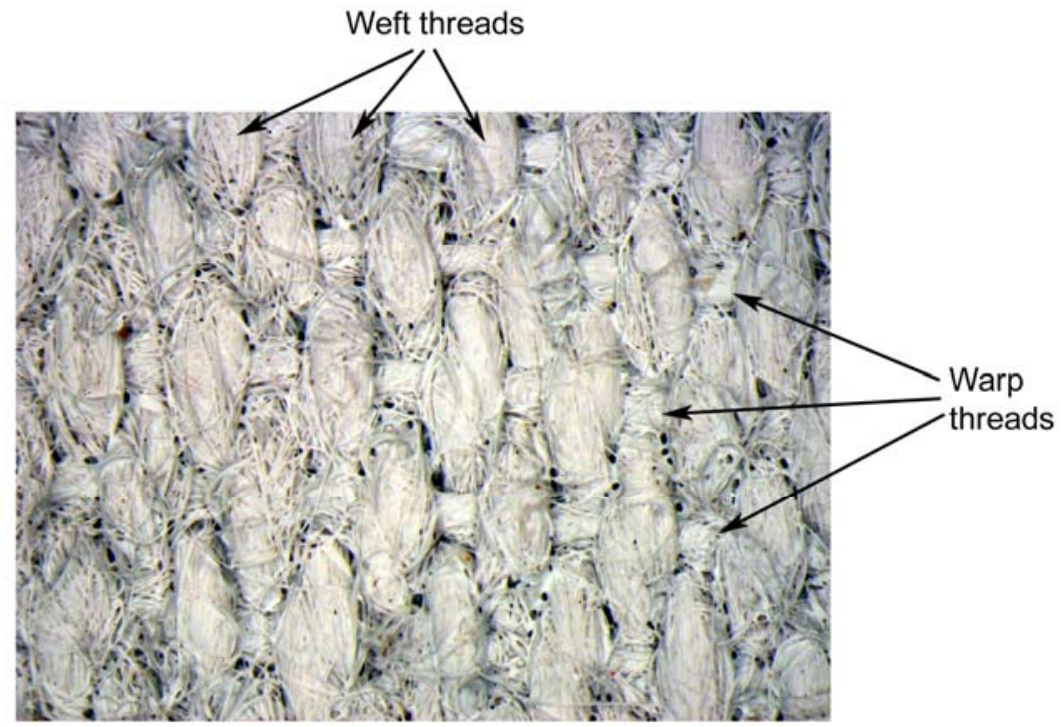

Fig.4: Microscopic image of tarp sample 1, where the warp threads are horizontal and weft threads are vertical 


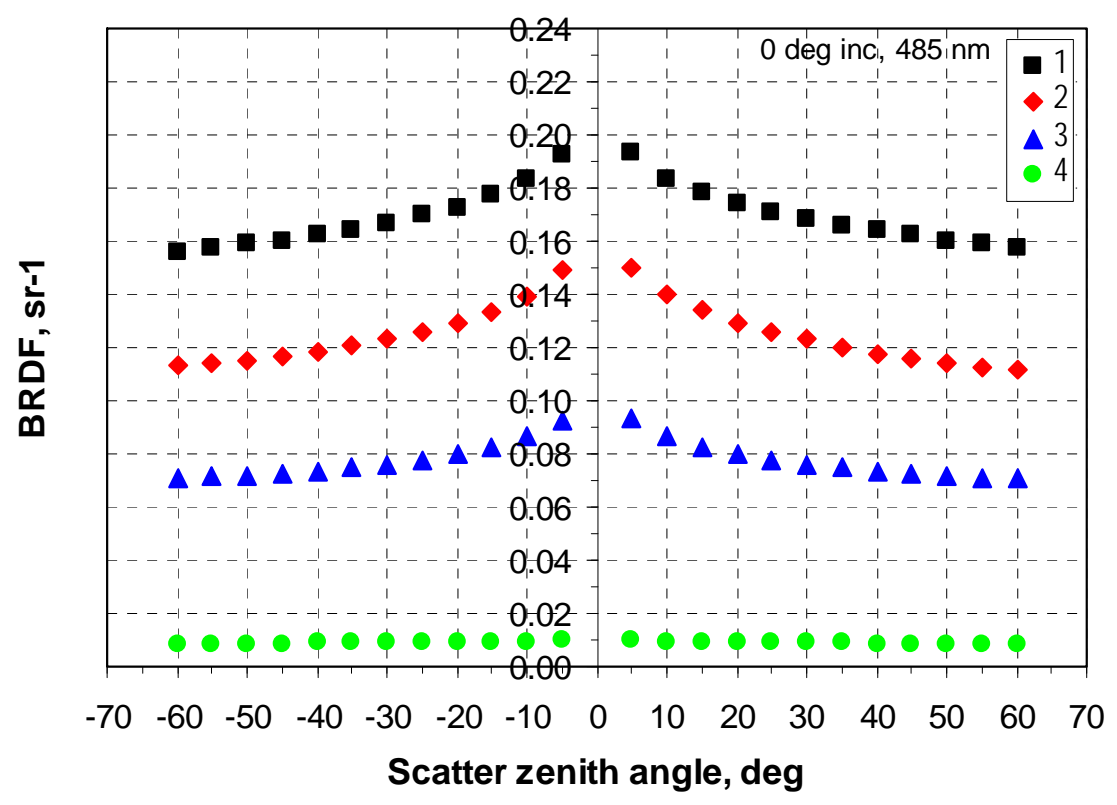

Fig.5: In-plane BRDF of tarps 1 to 4 at normal incidence, $485 \mathrm{~nm}, 0^{\circ}$ and $180^{\circ}$ scatter azimuth 


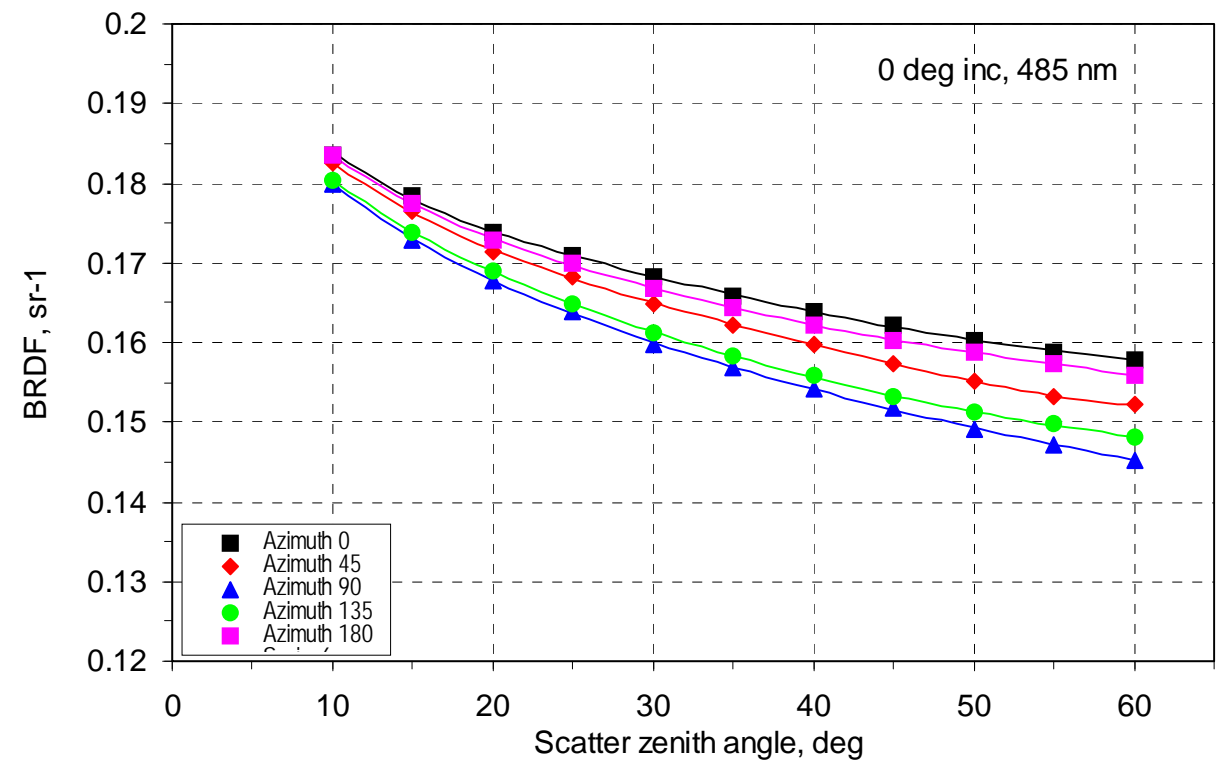

Fig.6: BRDF of tarp 1 at normal incidence at different scatter azimuth angles, $485 \mathrm{~nm}$, fitting and experimental points 


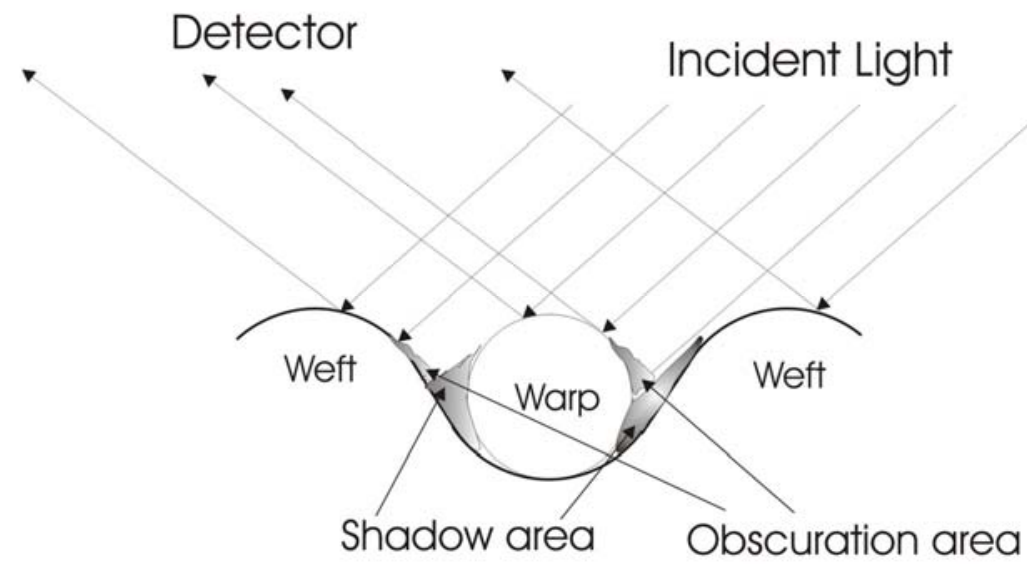

Fig.7: Shadowing and obscuration effects of tarps 


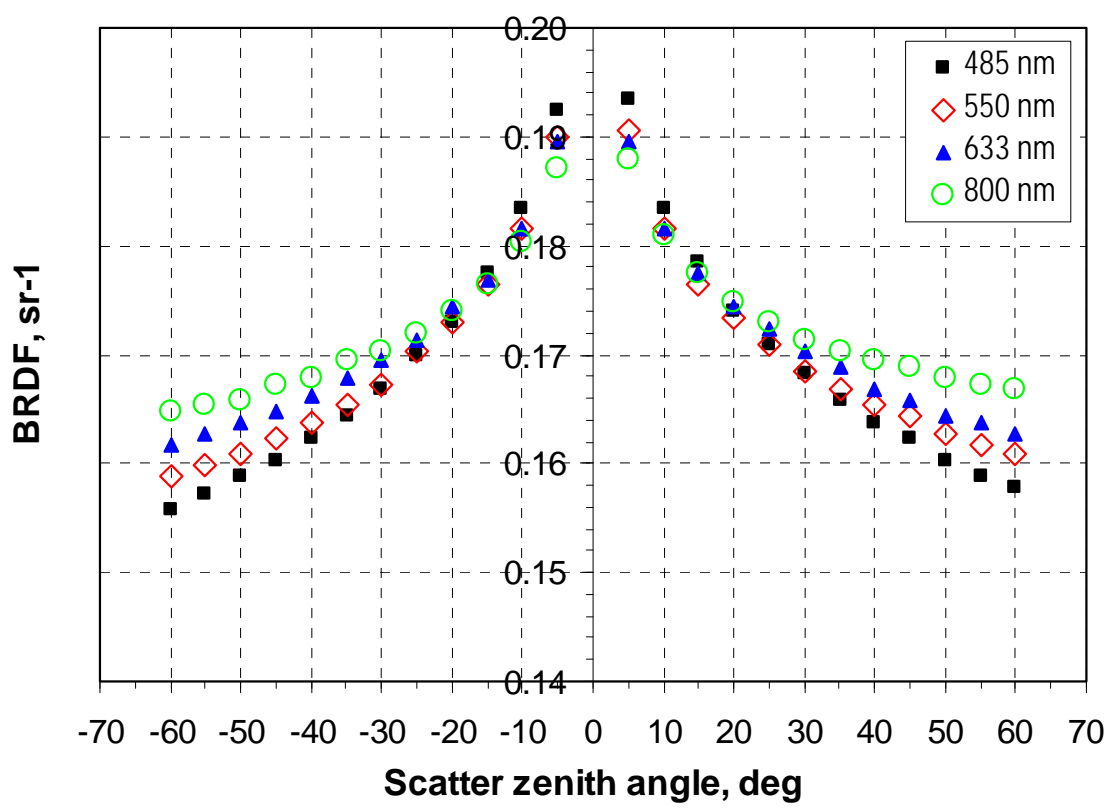

Fig.8: BRDF of tarp 1 at normal incidence, $485,550,633$, and $800 \mathrm{~nm}, 0^{\circ}$ and $180^{\circ}$ scatter azimuth angles 


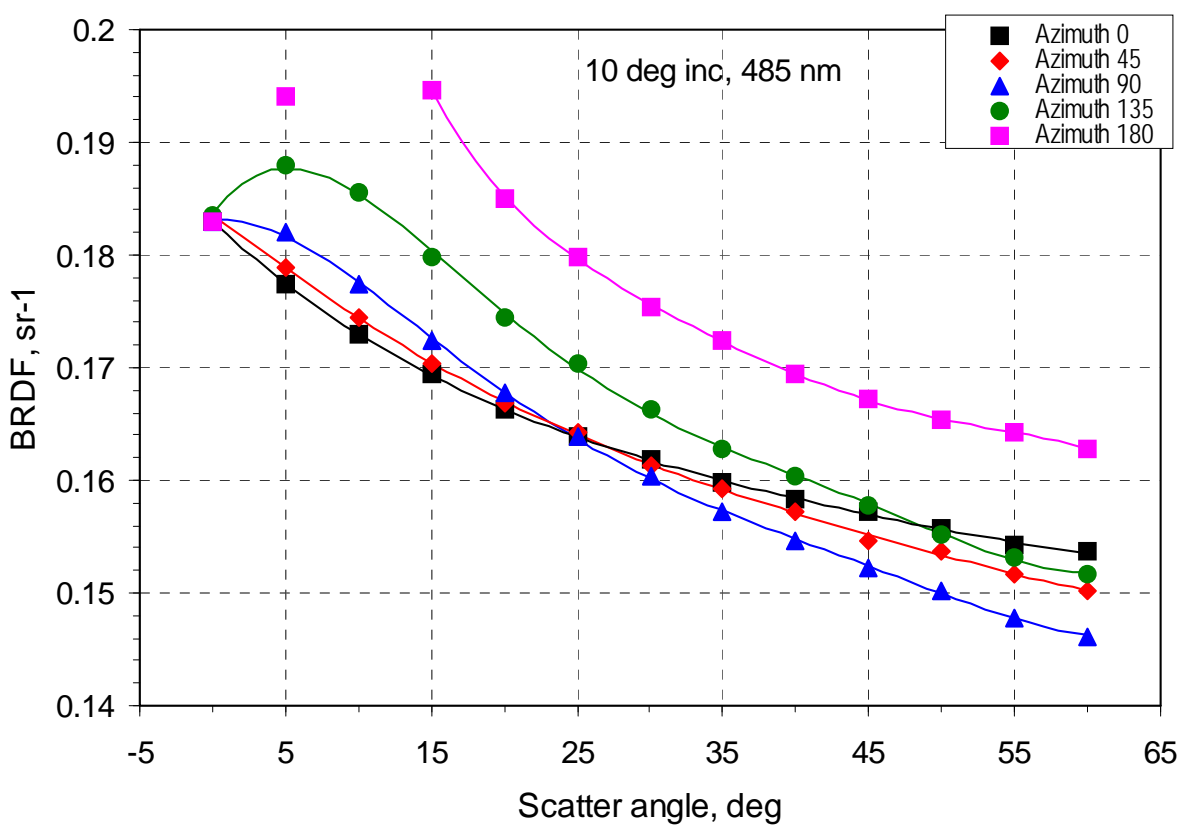

Fig.9: BRDF of tarp 1 at $10^{\circ}$ incidence at different scatter azimuth angles, $485 \mathrm{~nm}$, fitting and experimental points 


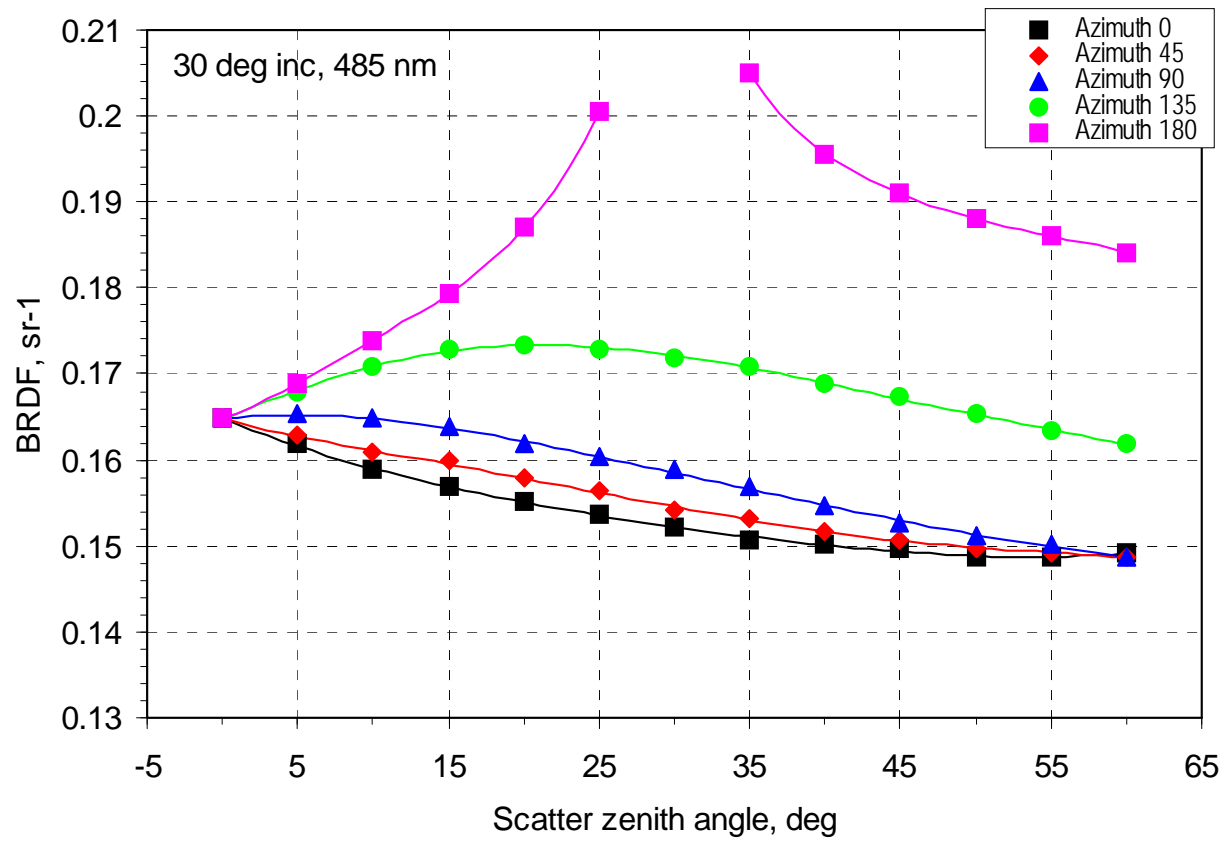

Fig.10: BRDF of tarp 1 at $30^{\circ}$ incidence at different scatter azimuth angles, $485 \mathrm{~nm}$, fitting and experimental points 


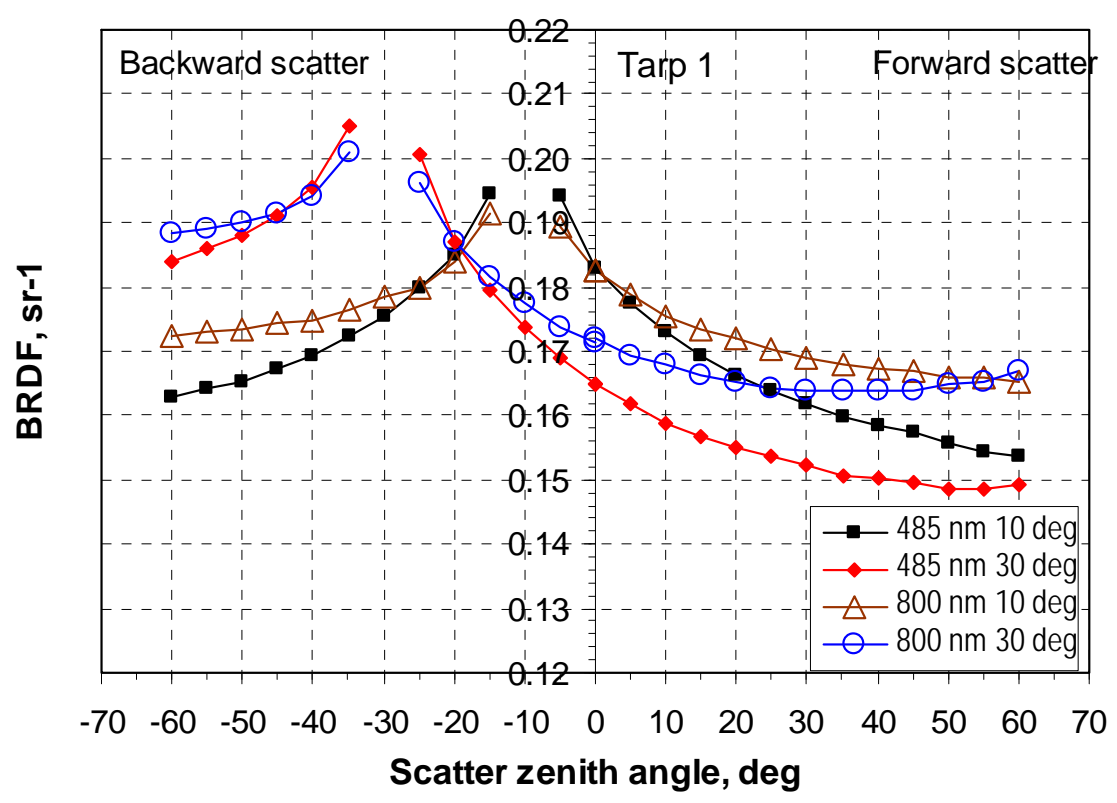

Fig.11: Forward - Backward scatter, tarp 1, 485 and $800 \mathrm{~nm}, 10^{\circ}$ and $30^{\circ}$ incident angles 


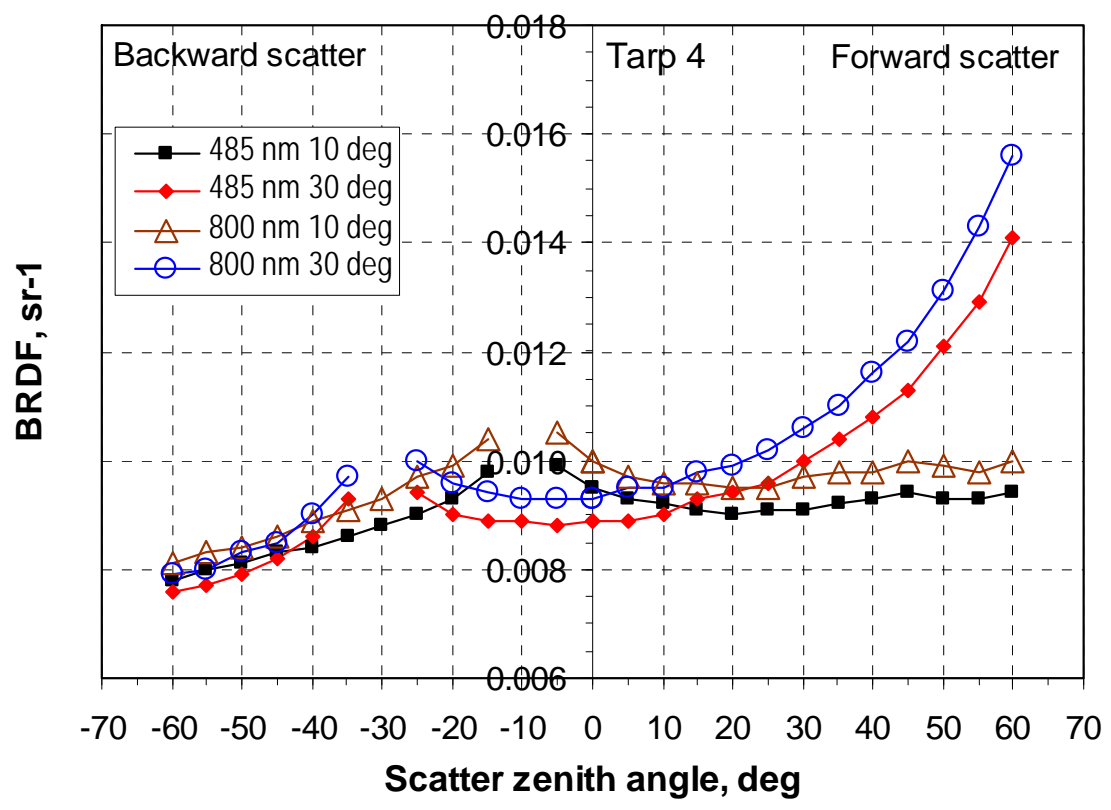

Fig.12: Forward - Backward scatter, tarp 4, 485 and $800 \mathrm{~nm}, 10^{\circ}$ and $30^{\circ}$ incident angle 


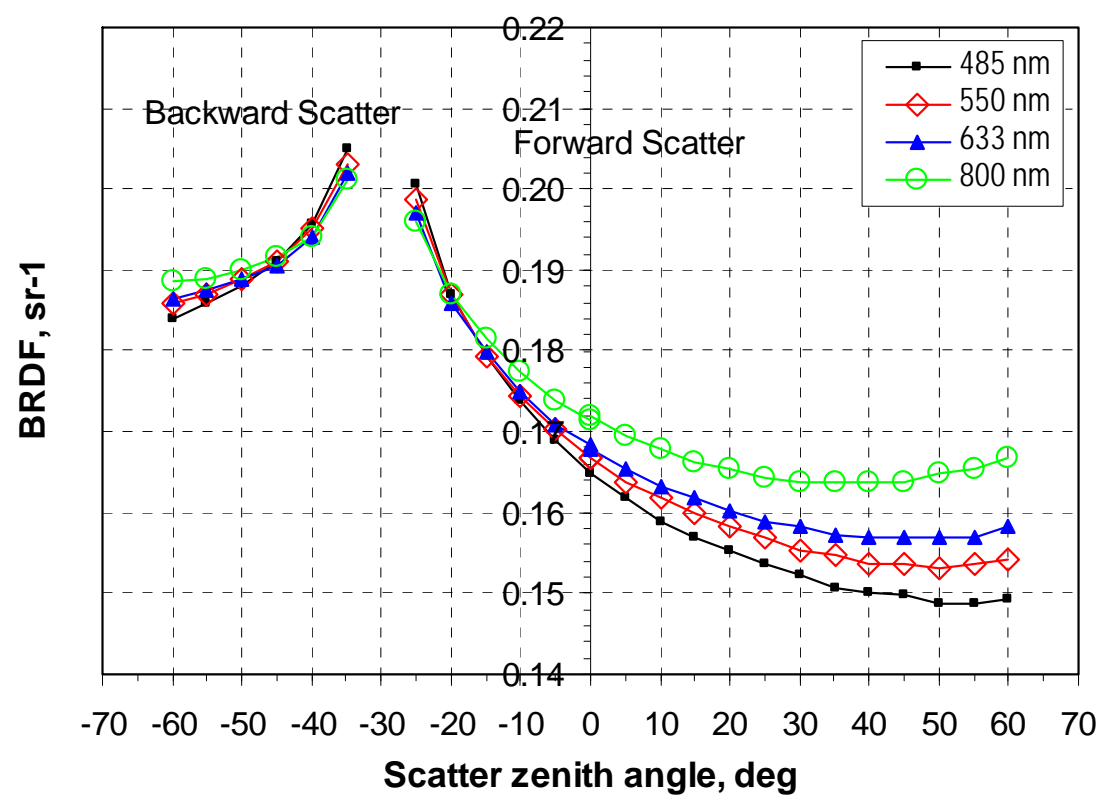

Fig.13: Tarp sample 1 at $30^{\circ}$ incidence, $485 \mathrm{~nm}, 550 \mathrm{~nm}, 633 \mathrm{~nm}$, and $800 \mathrm{~nm}$ 